Available at

www.ComputerScienceWeb.com

¿Direct.

\title{
Prioritised fuzzy constraint satisfaction problems: axioms, instantiation and validation
}

\author{
Xudong Luo ${ }^{\mathrm{a}, *}$, Jimmy Ho-man Lee ${ }^{\mathrm{b}}$, Ho-fung Leung ${ }^{\mathrm{b}}$, Nicholas R. Jennings ${ }^{\mathrm{a}}$ \\ ${ }^{a}$ Department of Electronics and Computer Science, The University of Southampton, \\ Highfield, Southampton SO17 1BJ, UK \\ ${ }^{\mathrm{b}}$ Department of Computer Science and Engineering, The Chinese University of Hong Kong, \\ Shatin, Hong Kong, People's Republic of China
}

Received 10 October 2000; received in revised form 29 July 2002; accepted 31 July 2002

\begin{abstract}
This paper identifies a generic axiom framework for prioritised fuzzy constraint satisfaction problems (PFCSPs), and proposes methods to instantiate it (i.e., to construct specific schemes which obey the generic axiom framework). In particular, we give five methods to construct the priority operators that are used for calculating the local satisfaction degree of a prioritised fuzzy constraint, and identify priority T-norm operators that can be used for calculating the global satisfaction degree of a prioritised fuzzy constraint problem. Moreover, a number of numerical examples and real examples are used to validate our system, and thus we further obtain some insights into our system. In addition, we explore the relationship between weight schemes and prioritised FCSP schemes, and reveal that the weighted FCSP schemes are the dual of prioritised FCSP schemes, which can, correspondingly, be called posterioritised FCSP schemes.
\end{abstract}

(c) 2002 Elsevier Science B.V. All rights reserved.

Keywords: Constraint satisfaction; Fuzzy set theory; Priority; Weight; Knowledge engineering; Non-monotonicity; Decision-making

\section{Introduction}

Many real-world problems (e.g., meeting scheduling [31,45,75], planning [61] and automated negotiation [35,73,4,39-41]) can be modelled as constraint satisfaction problems (CSPs) [51,74]. Solving a CSP involves finding an assignment of values to variables such that all its constraints hold. In

\footnotetext{
${ }^{*}$ Corresponding author. Tel.: +44-23-8059-3270.

E-mail address: x1@ecs.soton.ac.uk (X. Luo).
} 
the conventional framework for CSPs, constraints can never be violated. This is, however, sometimes inflexible. Thus, various efforts are made for equipping conventional CSP with soft constraints which can partially be violated. The extended frameworks include fuzzy CSPs $[63,83,57,17-64$, $11,19,20,23,37]$, probabilistic CSPs [27,2,59,60,72], and more general valued CSPs $[69,16,42,68]$ as well as semiring-based CSPs [8-10]. Among these extended frameworks, fuzzy CSPs are the most popular. Generally speaking, a crisp constraint can be viewed as a set of tuples, and thus using the concept of fuzzy sets a fuzzy constraint is regarded as a fuzzy set of tuples. Further, in order to deal with the different levels of importance of the different fuzzy constraints, Dubois et al. [19,20,22] introduce the concept of priorities into Fuzzy CSPs (FCSPs) to form prioritised FCSPs (PFCSPs).

This paper builds upon the work of Dubois et al. [19,20,23] and extends it in a number of directions. (1) To reveal axioms that the global satisfaction degree of a PFCSP should satisfy. Their work mainly reveals axioms that the local satisfaction degree of a prioritised constraint should fulfil (but, as we shall show, one of these properties is redundant). (2) To further discuss the construction of the schemes for PFCSPs, especially the construction of priority operators. Dubois et al. give one such scheme (hereafter the DFP scheme for short) in [19,20,23] and a full axiomatic discussion about possibilistic aggregation and more general Sugeno integrals in their other works. Moreover, they discuss different types of prioritised constraints (e.g., constraints with safeguards and conditional constraints). Our further discussion in this paper leads to some new results. (3) To distinguish the scale for priorities from the scale for constraint satisfaction degrees. The DFP scheme uses the same scale $[0,1]$ for constraint satisfaction degrees and priorities. This might lead to problems in some situations. (4) To clarify the relationship between prioritised FCSP schemes and weighted FCSP schemes. Traditionally, the concept of weight is used to indicate the importance level of an object among some objects [3,36,28,13-15], and so like the concept of priority it can also be used to indicate the importance level of a constraint among some constraints. Giving this, we clarify the difference between them.

We organise the study in this paper according to the principles of knowledge engineering [70]: when building a knowledge model, one first needs to describe the generic structure of knowledge, then instantiate this structure, and finally validate the knowledge model. Following this approach, this paper first introduces a generic axiom framework for PFCSPs. This is important because if we have such a framework we can hold commonalities in different specific schemes, develop the appropriate schemes for specific applications, and examine the relationships between the different schemes [46].

Having developed the generic framework, we then discuss the issue of how to instantiate it. That is, how to construct various specific schemes that satisfy the generic axiom system. These more specific schemes will offer more freedom in the selection of suitable schemes for particular applications. This is important because the best schemes are likely to vary from problem to problem. To this end, we present several construction methods and use these methods to develop several new schemes which are different from the one in [19,20,23]. In particular, we give five methods to construct the priority operators that are used for calculating the local satisfaction degree of a prioritised fuzzy constraint, and identify the priority T-norm operators that are used for calculating the global satisfaction degree of a prioritised fuzzy constraint problem. In addition, our results show that a PFCSP can be transformed equivalently into an FCSP, and so the techniques developed for solving FCSPs $[17,19,54,76,77]$ can be adopted for solving PFCSPs.

We then use numerical and real examples to validate our system. This reveals some deeper insights into our system. 
In addition, we explore the relationship between weighted FCSP schemes and prioritised FCSP schemes, and reveal that weighted FCSP schemes are the dual of prioritised FCSP schemes. Thus, in order to reflect the fact that the solutions of weighted FCSPs mainly depend on constraints with posteriority (relatively low importance levels), correspondingly to the prioritised FCSP schemes whose solutions mainly depend on constraints with priority (relatively high importance levels), weighted FCSP schemes can be called posterioritised FCSP schemes.

The rest of this paper is organised as follows. Section 2 recalls some concepts of FCSPs. Section 3 outlines our axiomatic framework for PFCSPs. Section 4 discusses the issue of instantiating the generic axiom framework. Section 5 validates our system. Section 6 clarifies the relationship between prioritised FCSP schemes and weighted FCSP schemes, and thus introduces the concept of posterioritised FCSPs. Section 7 compares our work with that of Dubois et al. in the field. The final section summaries our contributions and sheds light on the future direction of research.

\section{Preliminaries}

This section recalls some basic concepts and notations related to and FCSPs [83,57,11,19,20,23] which will be used throughout the paper.

Definition 1. A fuzzy constraint satisfaction problem (FCSP) is defined as a 3-tuple $\left(X, D, C^{f}\right)$, where:

(1) $X=\left\{x_{i} \mid i=1, \ldots, n\right\}$ is a finite set of variables.

(2) $D=\left\{d_{i} \mid i=1, \ldots, n\right\}$ is the set of domains. Each domain $d_{i}$ is a finite set containing the possible values for the corresponding variable $x_{i}$ in $X$.

(3) $C^{f}$ is a set of fuzzy constraints. That is,

$$
C^{f}=\left\{R_{i}^{f} \mid \mu_{R_{i}^{f}}:\left(\prod_{x_{j} \in \operatorname{var}\left(R_{i}^{f}\right)} d_{j}\right) \rightarrow[0,1], i=1, \ldots, m\right\},
$$

where $\operatorname{var}\left(R_{i}^{f}\right)$ denotes the set of variables of fuzzy constraint $R_{i}^{f}$.

Definition 2. A label of a variable $x$ is an assignment of a value to the variable, denoted as $v_{x}$. A compound label $v_{X^{\prime}}$ of all variables in set $X^{\prime}=\left\{x_{1}^{\prime}, \ldots, x_{m}^{\prime}\right\} \subseteq X$ is a simultaneous assignment of values to all variables in set $X^{\prime}$, that is,

$$
v_{X^{\prime}}=\left(v_{x_{1}^{\prime}}, \ldots, v_{x_{m}^{\prime}}\right) .
$$

The membership degree of a fuzzy constraint just indicates the local degree to which the constraint is locally satisfied with a compound label, and so the degree is also called the local satisfaction degree of the constraint for a compound label. The following definition gives the concept of global degree to which all constraints are satisfied with a compound label. 
Definition 3. In an FCSP $\left(X, D, C^{f}\right)$, given a compound label $v_{X}$ of all variables in $X$, the global satisfaction degree of the PFCSP for the compound label $v_{X}$ is defined as

$$
\alpha\left(v_{X}\right)=\oplus\left\{\mu_{R^{f}}\left(v_{\operatorname{var}\left(R^{f}\right)}\right) \mid R^{f} \in C^{f}\right\},
$$

where $\oplus$ is an aggregation from $[0,1]^{n}$ to $[0,1]$. A solution to an $\operatorname{FCSP}\left(X, D, C^{f}\right)$ is a compound label $v_{X}$ of all the variables in $X$ such that

$$
\alpha\left(v_{X}\right) \geqslant \alpha_{0},
$$

where $\alpha_{0} \in[0,1]$, called the FCSP's solution threshold, is predetermined.

Intuitively, solving an FCSP involves finding a compound label of all variables such that the constraints involved are satisfied, to some extent, with the compound label.

In the first paper about fuzzy set theory [81], Zadeh proposes to use $\min$ as $\oplus$ in the generic FCSP global satisfaction degree formula (3), that is,

$$
\alpha\left(v_{X}\right)=\min \left\{\mu_{R^{f}}\left(v_{\operatorname{var}\left(R^{f}\right)}\right) \mid R^{f} \in C^{f}\right\} .
$$

Bellman and Zadeh [6] later coined $\oplus$ as the confluence of constraints, acquiring different meanings in different cases. From a similar point of view, Zimmermann [84] points out that the choice of an appropriate aggregation operator largely depends on the context of the problem one deals with. Generally speaking,

(1) if one wants to find out the degree to which a compound label satisfies all constraints, a T-norm $\triangle$ could be used as $\oplus$ in (3), that is,

$$
\alpha\left(v_{X}\right)=\triangle\left\{\mu_{R^{f}}\left(v_{\operatorname{var}\left(R^{f}\right)}\right) \mid R^{f} \in C^{f}\right\}
$$

(2) if one intends to get the degree to which a compound label satisfies at least one of constraints, a T-conorm $\nabla$ could be used as $\oplus$ in (3), that is,

$$
\alpha\left(v_{X}\right)=\nabla\left\{\mu_{R^{f}}\left(v_{\operatorname{var}\left(R^{f}\right)}\right) \mid R^{f} \in C^{f}\right\},
$$

where T-norm and T-conorm are defined as below [71,5,21].

Definition 4. If an operator $\circ:[0,1] \times[0,1] \longrightarrow[0,1]$ satisfies:

(1) commutativity: $\forall a_{1}, a_{2} \in[0,1], a_{1} \circ a_{2}=a_{2} \circ a_{1}$,

(2) associativity: $\forall a_{1}, a_{2}, a_{3} \in[0,1],\left(a_{1} \circ a_{2}\right) \circ a_{3}=a_{1} \circ\left(a_{2} \circ a_{3}\right)$,

(3) monotonicity: $\forall a_{1}, a_{1}^{\prime}, a_{2}, a_{2}^{\prime} \in[0,1], a_{1} \leqslant a_{1}^{\prime} \wedge a_{2} \leqslant a_{2}^{\prime} \Rightarrow a_{1} \circ a_{2} \leqslant a_{1}^{\prime} \circ a_{2}^{\prime}$,

(4) boundary condition: $\forall a \in[0,1], a \circ 1=a$,

then $\circ$ is a Triangular norm (T-norm) on $[0,1]$, denoted as $\triangle$. If $\circ$ satisfies (1)-(3) and

(4) $)^{\prime}$ boundary condition: $\forall a \in[0,1], a \circ 0=a$,

then $\circ$ is a Triangular conorm (T-conorm) on $[0,1]$, denoted as $\nabla$. 
Two of the most important properties of $\triangle$ and $\nabla$ are as follows:

Lemma 1. $\forall a_{1}, \ldots, a_{n} \in[0,1]$,

$$
\triangle\left(a_{1}, \ldots, a_{n}\right) \leqslant \min \left\{a_{1}, \ldots, a_{n}\right\} \leqslant \max \left\{a_{1}, \ldots, a_{n}\right\} \leqslant \nabla\left(a_{1}, \ldots, a_{n}\right) .
$$

Lemma 2. $\forall a \in[0,1]$,

$$
\begin{aligned}
& a \triangle 0=0, \\
& a \nabla 1=1 .
\end{aligned}
$$

Example 1. Some examples of T-norms and T-conorms are listed as below.

(1) Zadeh operators $(\wedge, \vee)$ :

$$
\begin{aligned}
& a_{1} \wedge a_{2}=\min \left\{a_{1}, a_{2}\right\}, \\
& a_{1} \vee a_{2}=\max \left\{a_{1}, a_{2}\right\} .
\end{aligned}
$$

(2) Probability operators $(\bullet, \hat{+})$ :

$$
\begin{aligned}
& a_{1} \bullet a_{2}=a_{1} a_{2}, \\
& a_{1} \hat{+} a_{2}=a_{1}+a_{2}-a_{1} a_{2} .
\end{aligned}
$$

(3) Einstein operators $(\dot{E}, \stackrel{+}{E})$ :

$$
\begin{aligned}
a_{1} \dot{E} a_{2} & =\frac{a_{1} a_{2}}{1+\left(1-a_{1}\right)\left(1-a_{2}\right)}, \\
a_{1} \stackrel{+}{E} a_{2} & =\frac{a_{1}+a_{2}}{1+a_{1} a_{2}} .
\end{aligned}
$$

(4) Boundary operators $(\odot, \oplus)$ :

$$
\begin{aligned}
& a_{1} \odot a_{2}=\max \left\{0, a_{1}+a_{2}-1\right\}, \\
& a_{1} \oplus a_{2}=\min \left\{1, a_{1}+a_{2}\right\} .
\end{aligned}
$$

With these basics in place, the next section details our framework for PFCSPs.

\section{An axiomatic framework for PFCSPs}

In an FCSP, each constraint has no priority or, equivalently, all constraints have the same level of priority. This is not always true in practice. This section identifies an axiomatic framework for PFCSPs. 
Definition 5. A prioritised fuzzy constraint satisfaction problem (PFCSP) is a 4-tuple $\left(X, D, C^{f}, \rho\right)$, where $\left(X, D, C^{f}\right)$ is an FCSP, called the counterpart FCSP of the PFCSP, and $\rho: C^{f} \rightarrow[0, \infty)$ is a priority function.

Definition 6. In a PFCSP $\left(X, D, C^{f}, \rho\right)$, given a compound label $v_{X}$ of all variables in $X, \alpha_{\rho}\left(v_{X}\right)$, which is given by

$$
\alpha_{\rho}\left(v_{X}\right)=\bigoplus_{\rho}\left\{g\left(\rho\left(R^{f}\right), \mu_{R^{f}}\left(v_{\operatorname{var}\left(R^{f}\right)}\right)\right) \mid R^{f} \in C^{f}\right\}
$$

where $\bigoplus_{\rho}:[0,1]^{n} \rightarrow[0,1]$ and $g:[0,+\infty) \times[0,1] \rightarrow[0,1]$, is said to be the global satisfaction degree if the following properties are satisfied:

(1) If for the fuzzy constraint $R_{\max }^{f}, \rho_{\max }=\rho\left(R_{\max }^{f}\right)=\max \left\{\rho\left(R^{f}\right) \mid R^{f} \in C^{f}\right\}$ (hereafter unless otherwise specified, the symbols $R_{\max }^{f}$ and $\rho_{\max }$ always take the meaning here), then

$$
\mu_{R_{\max }^{f}}\left(v_{\operatorname{var}\left(R_{\max }^{f}\right)}\right)=0 \Rightarrow \alpha_{\rho}\left(v_{X}\right)=0 .
$$

(2) If $\exists \rho_{0} \in[0,1], \forall R^{f} \in C^{f}, \rho\left(R^{f}\right)=\rho_{0}$, then

$$
\alpha_{\rho}\left(v_{X}\right)=\triangle\left\{\mu_{R^{f}}\left(v_{\operatorname{var}\left(R^{f}\right)}\right) \mid R^{f} \in C^{f}\right\} .
$$

(3) For $R_{i}^{f}, R_{j}^{f} \in C^{f}$, suppose $\rho\left(R_{i}^{f}\right) \geqslant \rho\left(R_{j}^{f}\right)$, and there are two different compound labels $v_{X}$ and $v_{X}^{\prime}$ such that $\forall R^{f} \in C^{f}$,
(a) when $R^{f} \neq R_{i}^{f}$ and $R^{f} \neq R_{j}^{f}, \mu_{R^{f}}\left(v_{\operatorname{var}\left(R^{f}\right)}\right)=\mu_{R^{f}}\left(v_{\operatorname{var}\left(R^{f}\right)}^{\prime}\right)$,
(b) when $R^{f}=R_{i}^{f}, \mu_{R^{f}}\left(v_{\operatorname{var}\left(R^{f}\right)}\right)=\mu_{R^{f}}\left(v_{\operatorname{var}\left(R^{f}\right)}^{\prime}\right)+\delta$,
(c) when $R^{f}=R_{j}^{f}, \mu_{R^{f}}\left(v_{\operatorname{var}\left(R^{f}\right)}^{\prime}\right)=\mu_{R^{f}}\left(v_{\operatorname{var}\left(R^{f}\right)}\right)+\delta$.

If

$$
g\left(\rho\left(R_{i}^{f}\right), \mu_{R_{i}^{f}}\left(v_{\operatorname{var}\left(R_{i}^{f}\right)}\right)\right) \leqslant g\left(\rho\left(R_{j}^{f}\right), \mu_{R_{j}^{f}}\left(v_{\operatorname{var}\left(R_{j}^{f}\right)}\right)\right),
$$

then

$$
\alpha_{\rho}\left(v_{X}\right) \geqslant \alpha_{\rho}\left(v_{X}^{\prime}\right) .
$$

(4) For two different compound labels $v_{X}$ and $v_{X}^{\prime}$, if $\forall R^{f} \in C^{f}$,

$$
\mu_{R^{f}}\left(v_{\operatorname{var}\left(R^{f}\right)}\right) \geqslant \mu_{R^{f}}\left(v_{\operatorname{var}\left(R^{f}\right)}^{\prime}\right),
$$

then

$$
\left.\alpha_{\rho}\left(v_{X}\right) \geqslant \alpha_{\rho}\left(v_{X}^{\prime}\right)\right)
$$

(5) If there exists a compound labels $v_{X}$ such that

$$
\forall R^{f} \in C^{f}, \mu_{R^{f}}\left(v_{\operatorname{var}\left(R^{f}\right)}\right)=1,
$$

then

$$
\alpha_{\rho}\left(v_{X}\right)=1
$$


The above definition can be viewed as giving five axioms which the generic PFCSP global satisfaction degree formula (19) should obey. The first and third axioms are critical. We explain these first before moving onto others.

The first axiom means that in a PFCSP, although fuzzy constraints can be partially violated by a variable assignment, it is absolutely impossible for a variable assignment to be accepted as a solution if one of the most important fuzzy constraints is completely violated by the variable assignment. This mirrors the situation that can occur reasonably often in the real-world. The following are some examples (a more formal example is given in Section 5.2).

Example 2. (1) In the United Nations, if a proposal is not approved by one of the most important countries, i.e., USA, UK, France, China and Russia, this proposal cannot be passed even if all other countries agree to it.

(2) Suppose members of a research group want to meet weekly for 2 hours. This group consists of two supervisors, one post-doctoral research fellow, two Ph.D. students, two master students, and two undergraduate students for final year projects. All of them are very busy. Assume that their timetables, constraints and preferences are modelled by fuzzy constraints [45]. Clearly, the two supervisors are the most important people in the group, and so their constraints should be the most important and assigned the highest priority. Then, the priorities assigned to the constraints of the post-doctoral research fellow, the Ph.D. students, the master students and the undergraduate students are decreasing. Clearly, if an acceptable time slot can be found for each group member apart from the two supervisors (because, for example, during the time slot they are teaching in the classrooms), the group cannot have a meeting at that time.

(3) Suppose a researcher wants to organise his travel from Hong Kong to Berlin for an international conference. He has several constraints. The most important ones are: his wife demands him to visit her mother in London and he must be in Berlin on the day he presents his paper. Clearly, as long as these two constraints cannot be satisfied, even if a travel agent can offer him a three night free accommodation in Berlin plus a budget ticket, he cannot accept the offer.

The third axiom captures the essential meaning of priorities: if one wants to raise the global satisfaction degree of all prioritised constraints, a constraint with a relatively high priority must be sufficiently satisfied prior to a constraint with a relatively low priority. This is accordance with the meaning of the word priority in English dictionaries (e.g., [58]). In fact, the concept of priority has the following characteristics:

- it measures the relative importance among things in a group to determine only their relative precedence, and

- the higher the priority of one thing, the earlier the thing should be handled or the more preferred is the thing.

Consequently, the higher the priority of a constraint, the more preference satisfying the constraint should be given when finding a solution. That is precisely the reason why this kind of framework is called prioritised.

The second axiom reveals that since priorities are relative, their effect should disappear when they are the same. The fourth axiom captures the monotonicity of the aggregation operation: the 
global satisfaction degree of all prioritised fuzzy constraints in a PFCSP should increase when the local satisfaction degrees of all corresponding non-prioritised constraints increase. The fifth axiom means that when each corresponding non-prioritised constraint is locally satisfied completely with a compound label, there is no reason why the global satisfaction degree of all constraints in a PFCSP should not be 1 . In addition, the generic PFCSP global satisfaction degree formula (19) implies that the result of an aggregation should not be affected by the aggregation ordering.

Definition 7. A solution to a PFCSP $\left(X, D, C^{f}, \rho\right)$ is a compound label $v_{X}$ of all variables in $X$ such that

$$
\alpha_{\rho}\left(v_{X}\right) \geqslant \alpha_{0}
$$

where $\alpha_{0} \in[0,1]$, called the PFCSP's solution threshold, is predetermined.

\section{Axiom instantiation}

The generic axiom framework, proposed in the previous section, is a skeletal form of a scheme for PFCSPs. It can be instantiated for a class of specific schemes for PFCSPs. This section discusses the issue of constructing specific schemes which instantiate the generic axiom framework.

\subsection{Instantiation of $g$ function}

The generic PFCSP global satisfaction degree (19) actually outlines the common structure of various PFCSP global satisfaction degree formulae for aggregating operations on the local satisfaction degrees of all prioritised fuzzy constraints. Thus, when specifying (19), function $g$ should be specified first. Since the role of the priority of a fuzzy constraint $R^{f} \in C^{f}$ can be regarded as a prioritised factor to the local satisfaction degree of the constraint, $g\left(\mu_{R^{f}}\left(v_{\operatorname{var}\left(R^{f}\right)}\right), \rho\left(R^{f}\right)\right)$ can be viewed as the local satisfaction degree of the prioritised constraint, denoted as $\mu_{R^{f}}^{\rho}\left(v_{\operatorname{var}\left(R^{f}\right)}\right)$. Formally, we have:

Definition 8. In a PFCSP $\left(X, D, C^{f}, \rho\right)$, given a compound label $v_{X}$ of all variables in $X$, the local satisfaction degree of a prioritised fuzzy constraint $R^{f} \in C^{f}$ is given by

$$
\mu_{R^{f}}^{\rho}\left(v_{\operatorname{var}\left(R^{f}\right)}\right)=\left(\rho\left(R^{f}\right) \oslash \rho_{\max }\right) \diamond \mu_{R^{f}}\left(v_{\operatorname{var}\left(R^{f}\right)}\right),
$$

where operator $\oslash:[0, \infty) \times(0, \infty) \rightarrow[0,1]$, called a general division operator, satisfies:

(1) $\forall a \in(0, \infty), a \oslash a=1$,

(2) $\forall a \in(0, \infty), 0 \oslash a=0$,

(3) $\forall a_{1}, a_{1}^{\prime} \in[0, \infty), a_{2} \in(0, \infty), a_{1} \leqslant a_{1}^{\prime} \Rightarrow a_{1} \oslash a_{2} \leqslant a_{1}^{\prime} \oslash a_{2}$, and

(4) $\forall a_{1} \in[0, \infty), a_{2}, a_{2}^{\prime} \in(0, \infty), a_{2} \leqslant a_{2}^{\prime} \Rightarrow a_{1} \oslash a_{2} \geqslant a_{1}^{\prime} \oslash a_{2}$;

and operator $\diamond:[0,1] \times[0,1] \rightarrow[0,1]$, called a priority operator, satisfies:

(1) $\forall a_{1}, a_{2}, a_{2}^{\prime} \in[0,1], a_{2} \leqslant a_{2}^{\prime} \Rightarrow a_{1} \diamond a_{2} \leqslant a_{1} \diamond a_{2}^{\prime}$, 
(2) $\forall a_{1}, a_{1}^{\prime}, a_{2} \in[0,1], a_{1} \leqslant a_{1}^{\prime} \Rightarrow a_{1} \diamond a_{2} \geqslant a_{1}^{\prime} \diamond a_{2}$,

(3) $\forall a \in[0,1], 1 \diamond a=a$, and

(4) $\forall a \in[0,1], 0 \diamond a=1$.

The axioms on general division operators capture the basic idea behind the usual arithmetic division operator. This operator and the parameter $\rho_{\max }$ are critical to ensuring the first two axioms in Definition 6 are satisfied (we shall see later). The following are two examples of such general division operators.

Example 3. It is easy to check that operators $\oslash_{1}$ and $\oslash_{2}$, defined as follows, satisfy the axioms of general division operators:

$$
\begin{aligned}
& a_{1} \oslash_{1} a_{2}=\frac{a_{1}}{a_{2}} ; \\
& a_{1} \oslash_{2} a_{2}= \begin{cases}c^{a_{2}-a_{1}} & \text { if } a_{1} \neq 0, \\
0 & \text { otherwise, }\end{cases}
\end{aligned}
$$

where $c \in(0,1)$ is a constant.

The four axioms about priority operators capture the following intuitions. ${ }^{1}$ (1) The local satisfaction degree of a prioritised constraint should increase with that of its non-prioritised counterpart. (2) Given the local satisfaction degree of a constraint, the satisfaction degree of the corresponding prioritised constraint decreases when the priority increases. So, the higher the priority of a constraint, the more sufficiently the constraint should be satisfied if we want to get a solution with a higher global satisfaction degree. (3) When a constraint has the priority of $100 \%$, namely it has the relatively highest priority, its prioritised satisfaction degree is equal to its non-prioritised counterpart. (4) When a constraint has the lowest priority 0 , it means that it is immaterial whether the constraint is satisfied with a compound label. Accordingly, we can treat it as being satisfied with an arbitrary compound label. That is, the local satisfaction degree of the prioritised constraint should be 1 .

The following theorem for constructing priority operators is inspired by [23].

Theorem 1. Operator $\circ:[0,1] \times[0,1] \rightarrow[0,1]$, defined as

$$
a_{1} \circ a_{2}=\left(1-a_{1}\right) \nabla a_{2},
$$

is a priority operator.

Proof. Operator $\circ$, given by (31), satisfies the axioms of priority operators. In fact, by Definition 4 and Lemma 2 we have

$$
1 \geqslant a_{2} \geqslant a_{2}^{\prime} \geqslant 0 \Rightarrow\left(1-a_{1}\right) \nabla a_{2} \geqslant\left(1-a_{1}\right) \nabla a_{2}^{\prime} \Rightarrow a_{1} \circ a_{2} \geqslant a_{1} \circ a_{2}^{\prime},
$$

\footnotetext{
${ }^{1}$ The four axioms about priority operators are proposed by Dubois and Prade in [23] (see its second paragraph in p. 50 ), but they also put in one more axiom for priority operators, which is unnecessary. We will give a more detailed discussion about this in Section 7.1.
} 


$$
\begin{aligned}
& 1 \geqslant a_{1} \geqslant a_{1}^{\prime} \geqslant 0 \Rightarrow\left(1-a_{1}\right) \nabla a_{2} \leqslant\left(1-a_{1}^{\prime}\right) \nabla a_{2} \Rightarrow a_{1} \circ a_{2} \leqslant a_{1}^{\prime} \circ a_{2}, \\
& 1 \circ a=(1-1) \nabla a=0 \nabla a=a, \\
& 0 \circ a=(1-0) \nabla a=1 \nabla a=1 .
\end{aligned}
$$

The following example uses the above theorem to instantiate the generic prioritised fuzzy constraint satisfaction degree formula (28).

Example 4. Let operator $\oslash$ be given by (29), then by Theorem 1, the generic prioritised fuzzy constraint satisfaction degree formula (28) can be instantiated as

$$
\mu_{R^{f}}^{\rho}\left(v_{\operatorname{var}\left(R^{f}\right)}\right)=\max \left\{1-\frac{\rho\left(R^{f}\right)}{\rho_{\max }}, \mu_{R^{f}}\left(v_{\operatorname{var}\left(R^{f}\right)}\right)\right\} .
$$

Let $\oslash$ be given by (30), then by Theorem 1, the generic prioritised fuzzy constraint satisfaction degree formula (28) can be instantiated as

$$
\mu_{R^{f}}^{\rho}\left(v_{v a r\left(R^{f}\right)}\right)= \begin{cases}\max \left\{1-c^{\rho_{\max }-\rho\left(R^{f}\right)}, \mu_{R^{f}}\left(v_{v a r\left(R^{f}\right)}\right)\right\} & \text { if } \rho\left(R^{f}\right) \neq 0, \\ 1 & \text { otherwise, }\end{cases}
$$

where $c \in(0,1)$ is a constant.

The following theorem shows a number of other ways to construct new priority operators from existing operators. We believe it is significant that a method is able to reuse the existing functions and operators to construct something new. In fact, in software and knowledge engineering (see [56, p. 11]), the reuse of significant parts of developed systems, rather than building new systems from scratch, is beneficial as it helps to reduce the cost of building new application systems, and helps to define the space of problem solving methods.

Theorem 2. Let $S_{\diamond}$ be the set of all possible priority operators, and

$$
H=\left\{h:[0,1] \rightarrow[0,1] \mid x_{1} \geqslant x_{2} \Leftrightarrow h\left(x_{1}\right) \geqslant h\left(x_{2}\right), h(0)=0, h(1)=1\right\} .
$$

Then $\forall a_{1}, a_{2} \in[0,1], h, h_{1}, h_{2} \in H, \diamond, \diamond_{1}, \diamond_{2} \in S_{\diamond}$, the following operators are in $S_{\diamond}$ :

$$
\begin{aligned}
& a_{1} \circ_{1} a_{2}=\left(1-h\left(a_{1}\right)\right) \nabla a_{2}, \\
& a_{1} \circ_{2} a_{2}=h\left(a_{1}\right) \diamond a_{2}, \\
& a_{1} \circ_{3} a_{2}=h_{1}\left(a_{1}\right) \diamond_{1}\left(h_{2}\left(a_{1}\right) \diamond_{2} a_{2}\right) .
\end{aligned}
$$

Proof. Operators $\circ_{1}, \circ_{2}$ and $\circ_{3}$ are checked one by one, as follows:

(1) For operator $\circ_{1}$, by Definition 4 and Lemma 2, we have

$$
\begin{aligned}
1 \geqslant a_{2} \geqslant a_{2}^{\prime} \geqslant 0 & \Rightarrow\left(1-h\left(a_{1}\right)\right) \nabla a_{2} \geqslant\left(1-h\left(a_{1}\right)\right) \nabla a_{2}^{\prime} \\
& \Rightarrow a_{1} \circ_{1} a_{2} \geqslant a_{1} \circ_{1} a_{2}^{\prime},
\end{aligned}
$$




$$
\begin{aligned}
1 \geqslant a_{1} \geqslant a_{1}^{\prime} \geqslant 0 & \Rightarrow h\left(a_{1}\right) \geqslant h\left(a_{1}^{\prime}\right) \\
& \Rightarrow 1-h\left(a_{1}\right) \leqslant 1-h\left(a_{1}^{\prime}\right) \\
& \Rightarrow\left(1-h\left(a_{1}\right)\right) \nabla a_{2} \leqslant\left(1-h\left(a_{1}^{\prime}\right)\right) \nabla a_{2} \\
& \Rightarrow a_{1} \circ_{1} a_{2} \leqslant a_{1}^{\prime} \circ_{1} a_{2},
\end{aligned}
$$

$$
\begin{aligned}
& 1 \circ_{1} a=(1-h(1)) \nabla a=(1-1) \nabla a=0 \nabla a=a, \\
& 0 \circ_{1} a=(1-h(0)) \nabla a=(1-0) \nabla a=1 \nabla a=1 .
\end{aligned}
$$

(2) For operator $\mathrm{O}_{2}$, by Definition 4, we have

$$
\begin{aligned}
1 \geqslant a_{2} \geqslant a_{2}^{\prime} \geqslant 0 & \Rightarrow h\left(a_{1}\right) \diamond a_{2} \geqslant h\left(a_{1}\right) \diamond a_{2}^{\prime} \\
& \Rightarrow a_{1} \circ_{2} a_{2} \geqslant a_{1} \circ_{2} a_{2}^{\prime}, \\
1 \geqslant a_{1} \geqslant a_{1}^{\prime} \geqslant 0 & \Rightarrow h\left(a_{1}\right) \geqslant h\left(a_{1}^{\prime}\right) \\
& \Rightarrow h\left(a_{1}\right) \diamond a_{2} \leqslant h\left(a_{1}^{\prime}\right) \diamond a_{2} \\
& \Rightarrow a_{1} \circ_{2} a_{2} \leqslant a_{1}^{\prime} \circ_{2} a_{2},
\end{aligned}
$$

$$
\begin{aligned}
& 1 \circ_{2} a=h(1) \diamond a=1 \diamond a=a, \\
& 0 \circ_{2} a=h(0) \diamond a=0 \diamond a=1 .
\end{aligned}
$$

(3) For operator $\mathrm{o}_{3}$, by Definition 4 , we have

$$
\begin{aligned}
& 1 \geqslant a_{2} \geqslant a_{2}^{\prime} \geqslant 0 \Rightarrow h_{2}\left(a_{1}\right) \diamond_{2} a_{2} \geqslant h_{2}\left(a_{1}\right) \diamond_{2} a_{2}^{\prime} \\
& \Rightarrow h_{1}\left(a_{1}\right) \diamond_{1}\left(h_{2}\left(a_{1}\right) \diamond_{2} a_{2}\right) \geqslant h_{1}\left(a_{1}\right) \diamond_{1}\left(h_{2}\left(a_{1}\right) \diamond_{2} a_{2}^{\prime}\right) \\
& \Rightarrow a_{1} \diamond_{3} a_{2} \geqslant a_{1} \diamond_{3} a_{2}^{\prime}, \\
& 1 \geqslant a_{1} \geqslant a_{1}^{\prime} \geqslant 0 \Rightarrow h\left(a_{1}\right) \geqslant h\left(a_{1}^{\prime}\right) \\
& \Rightarrow\left\{\begin{array}{l}
h\left(a_{1}\right) \diamond_{1}\left(h\left(a_{1}\right) \diamond_{2} a_{2}\right) \leqslant h\left(a_{1}^{\prime}\right) \diamond_{1}\left(h\left(a_{1}\right) \diamond_{2} a_{2}\right) \\
h\left(a_{1}\right) \diamond_{2} a_{2} \leqslant h\left(a_{1}^{\prime}\right) \diamond_{2} a_{2}
\end{array}\right. \\
& \Rightarrow h\left(a_{1}\right) \diamond_{1}\left(h\left(a_{1}\right) \diamond_{2} a_{2}\right) \leqslant h\left(a_{1}^{\prime}\right) \diamond_{1}\left(h\left(a_{1}^{\prime}\right) \diamond_{2} a_{2}\right) \\
& \Rightarrow a_{1} \diamond_{3} a_{2} \leqslant a_{1} \diamond_{3} a_{2}^{\prime}, \\
& 1 \diamond_{3} a=h_{1}(1) \diamond_{1}\left(h_{2}(1) \diamond_{2} a\right)=1 \diamond_{1}\left(1 \diamond_{2} a\right)=1 \diamond_{2} a=a, \\
& 0 \diamond_{3} a=h_{1}(0) \diamond_{1}\left(h_{2}(0) \diamond_{2} a\right)=0 \diamond_{1}\left(0 \diamond_{2} a\right)=1 .
\end{aligned}
$$

Clearly, by Theorem 2, we can use functions in $H$ to construct more priority operators from the available priority operators (although we may not construct all priority operators by Theorem 2).

Example 5. Let $h_{1}(x)=\sin \frac{\pi}{2} x, h_{2}(x)=1-\cos \frac{\pi}{2} x, h_{3}(x)=\sqrt{x}, a_{1} \nabla_{1} a_{2}=\left(a_{1}+a_{2}\right) /$ $\left(1+a_{1} a_{2}\right)$, and $a_{1} \nabla_{2} a_{2}=a_{1}+a_{2}-a_{1} a_{2}$. Then, by Theorem 2 , the following operators are priority 
operators:

$$
\begin{aligned}
a_{1} \diamond_{1} a_{2} & =\left(1-h_{1}\left(a_{1}\right)\right) \nabla_{1} a_{2}=\frac{1-\sin \left(\frac{\pi}{2} a_{1}\right)+a_{2}}{1+\left(1-\sin \left(\frac{\pi}{2} a_{1}\right)\right) a_{2}}, \\
a_{1} \diamond_{2} a_{2} & =\left(1-h_{2}^{-}\left(a_{1}\right)\right) \nabla_{2} a_{2}=\cos \left(\frac{\pi}{2} a_{1}\right)+a_{2}-a_{2} \times \cos \left(\frac{\pi}{2} a_{1}\right), \\
a_{1} \diamond_{3} a_{2} & =h_{1}\left(a_{1}\right) \diamond_{1} a_{2}=\frac{1-\sin \left(\frac{\pi}{2} \sin \left(\frac{\pi}{2} a_{1}\right)\right)+a_{2}}{1+\left(1-\sin \left(\frac{\pi}{2} \sin \left(\frac{\pi}{2} a_{1}\right)\right)\right) a_{2}}, \\
a_{1} \diamond_{4} a_{2} & =h_{2}\left(a_{1}\right) \diamond_{2}\left(h_{1}\left(a_{1}\right) \diamond_{1} a_{2}\right) \\
& =\cos \left(\frac{\pi}{2} \sqrt{a_{1}}\right)+\frac{1-\sin \left(\frac{\pi}{2} \sin \left(\frac{\pi}{2} a_{1}\right)\right)+a_{2}}{1+\left(1-\sin \left(\frac{\pi}{2} \sin \left(\frac{\pi}{2} a_{1}\right)\right)\right) a_{2}}\left(1-\cos \left(\frac{\pi}{2} \sqrt{a_{1}}\right)\right) .
\end{aligned}
$$

The following theorem gives a beautiful priority operator directly.

Theorem 3. Operator $\circ:[0,1] \times[0,1] \rightarrow[0,1]$, defined as follows, is a priority operator:

$$
a_{1} \circ a_{2}=\left(a_{2}-1\right) a_{1}+1 \text {. }
$$

Proof. Operator $\circ$, given by (41), satisfies the axioms of priority operators. In fact,

$$
\begin{aligned}
& a_{2} \leqslant a_{2}^{\prime} \Rightarrow\left(a_{2}-1\right) a_{1}+1 \leqslant\left(a_{2}^{\prime}-1\right) a_{1}+1 \Rightarrow a_{1} \circ a_{2} \leqslant a_{1} \circ a_{2}^{\prime}, \\
& a_{1} \leqslant a_{1}^{\prime} \Rightarrow\left(a_{2}-1\right) a_{1}+1 \geqslant\left(a_{2}-1\right) a_{1}^{\prime}+1 \Rightarrow a_{1} \circ a_{2} \geqslant a_{1}^{\prime} \circ a_{2}, \\
& 1 \circ a_{2}=\left(a_{2}-1\right) a_{1}+1=\left(a_{2}-1\right) \times 1+1=a_{2}, \\
& 0 \circ a_{2}=\left(a_{2}-1\right) a_{1}+1=\left(a_{2}-1\right) \times 0+1=1 .
\end{aligned}
$$

\subsection{The instantiation of $\oplus_{\rho}$}

The previous subsection discussed the instantiation issue of the function $g$ in the generic PFCSP global satisfaction degree formula (19). Now, we turn to discuss the instantiation issue of $\oplus_{\rho}$ in (19).

Since a solution to a PFCSP usually needs to satisfy all constraints or the proposition $R_{1}^{f} \wedge \cdots \wedge R_{m}^{f}$ where each $R_{i}^{f} \in C^{f}(i=1, \ldots, m)$, we can define the function $\oplus_{\rho}$ as a T-norm $\triangle$ because in fuzzy set theory T-norm are extensively used to model logical connective $\wedge$ (and) [5]. Theorem 4 in the following guarantees that such a definition is reasonable according to our axiomatic framework proposed in Definition 6. However, in order to prove Theorem 4, T-norms should be restricted to the following subclass of T-norms: ${ }^{2}$

Definition 9. A T-norm $\triangle$ is a priority T-norm if under condition

$$
0 \leqslant a_{1} \leqslant a_{2} \wedge \delta>0 \wedge a_{1}+\delta \leqslant 1 \wedge a_{2}+\delta \leqslant 1
$$

\footnotetext{
${ }^{2}$ This will be seen clearly in the proof of Theorem 4 .
} 
the following inequality holds:

$$
\left(a_{1}+\delta\right) \triangle a_{2} \geqslant a_{1} \triangle\left(a_{2}+\delta\right) .
$$

Example 6. The following are examples of priority T-norms.

(1) Zadeh T-norm (11) is a priority T-norm. In fact, under condition (42) we have

$$
\min \left\{a_{1}+\delta, a_{2}\right\} \geqslant \min \left\{a_{1}, a_{2}\right\}=\min \left\{a_{1}, a_{2}+\delta\right\} .
$$

(2) Probability T-norm (13) is a priority T-norm. In fact, under condition (42), we have

$$
\left(a_{1}+\delta\right) a_{2}=a_{1} a_{2}+\delta a_{2} \geqslant a_{1} a_{2}+\delta a_{1}=a_{1}\left(a_{2}+\delta\right) .
$$

(3) Einstein T-norm (15) is a priority T-norm. In fact, under condition (42), we have

$$
\begin{aligned}
& a_{1}+\delta \leqslant 1 \wedge a_{2} \leqslant 1 \\
& \Rightarrow a_{1}+a_{2}+\delta \leqslant 2 \\
& \Rightarrow\left(a_{1}+a_{2}+\delta\right)\left(a_{1}-a_{2}\right) \geqslant 2\left(a_{1}-a_{2}\right) \\
& \Rightarrow 2 a_{2}-a_{2}^{2}-\delta a_{2} \geqslant 2 a_{1}-a_{2}^{2}-\delta a_{1} \\
& \Rightarrow 2 \delta a_{2}-\delta a_{2}^{2}-\delta^{2} a_{2} \geqslant 2 \delta a_{1}-\delta a_{2}^{2}-\delta^{2} a_{1} \\
& \Rightarrow a_{1} a_{2}\left(2-a_{1}-a_{2}+a_{1} a_{2}-\delta\right)+a_{1}^{2} a_{2} \delta \\
& \quad+2 \delta a_{2}-\delta a_{1} a_{2}-\delta a_{2}^{2}+a_{1} a_{2}^{2} \delta-\delta^{2} a_{2}+a_{1} a_{2} \delta \\
& \geqslant a_{1} a_{2}\left(2-a_{1}-a_{2}+a_{1} a_{2}-\delta\right)+a_{1} a_{2}^{2} \delta \\
& \quad+2 \delta a_{1}-\delta a_{1} a_{2}-\delta a_{1}^{2}+a_{1}^{2} a_{2} \delta-\delta^{2} a_{1}+a_{1} a_{2} \delta \\
& \Rightarrow \quad \frac{a_{1} a_{2}+\delta a_{2}}{2-a_{1}-a_{2}+a_{1} a_{2}-\delta+a_{2} \delta} \geqslant \frac{a_{1}}{2-a_{1}-a_{2}+a_{1} a_{2}-\delta+a_{1} \delta},
\end{aligned}
$$

that is,

$$
\frac{\left(a_{1}+\delta\right) a_{2}}{1+\left(1-\left(a_{1}+\delta\right)\right)\left(1-a_{2}\right)} \geqslant \frac{a_{1}\left(a_{2}+\delta\right)}{1+\left(1-a_{1}\right)\left(1-\left(a_{2}+\delta\right)\right)} .
$$

Before giving the main result in this subsection, we give a property of priority operators, which is useful in the proof of the main result.

Lemma 3. $\forall a \in[0,1]$,

$$
a \diamond 1=1 \text {. }
$$

Proof. By axioms 4, 2 and 3 of priority operators (which are listed in Definition 8), $\forall a \in[0,1]$, we have

$$
1=0 \diamond 1 \geqslant a \diamond 1 \geqslant 1 \diamond 1=1
$$


That is,

$$
\forall a \in[0,1], 1 \geqslant a \diamond 1 \geqslant 1 .
$$

Therefore, (44) holds.

Theorem 4. In a PFCSP $\left(X, D, C^{f}, \rho\right)$,

$$
\alpha_{\rho}\left(v_{X}\right)=\triangle\left\{\mu_{R^{f}}^{\rho}\left(v_{\operatorname{var}\left(R^{f}\right)}\right) \mid R^{f} \in C^{f}\right\}
$$

is the global satisfaction degree of the PFCSP for a compound label $v_{X}$.

Proof. (1) Let $\rho_{i}=\rho\left(R_{i}^{f}\right), i=1, \ldots, m$, and $\rho_{i_{0}}=\max \left\{\rho_{1}, \rho_{2}, \ldots, \rho_{m}\right\}$, if $\mu_{R_{i_{0}}^{f}}\left(v_{\operatorname{var}\left(R_{i_{0}}^{f}\right)}\right)=0$, we have

$$
\mu_{R_{i_{o}}^{f}}^{\rho}\left(v_{\operatorname{var}\left(R_{i_{o}}^{f}\right)}\right)=\left(\rho\left(R_{i_{o}}^{f}\right) \oslash \rho_{\max }\right) \diamond \mu_{R_{i_{o}}^{f}}\left(v_{\operatorname{var}\left(R_{i_{o}}^{f}\right)}\right)=\left(\rho_{0} \oslash \rho_{0}\right) \diamond 0=1 \diamond 0=0 .
$$

Thus, again noticing formula (9) and the commutativity of operator $\triangle$, we know the right-hand side of (45) is equal to 0 . So, the first axiom in Definition 6 is satisfied.

(2) If $\forall R^{f} \in C^{f}, \rho\left(R^{f}\right) \in C^{f}=\rho_{0}$, then we have

$$
\begin{aligned}
\mu_{R^{f}}^{\rho}\left(v_{\operatorname{var}\left(R^{f}\right)}\right) & =\left(\rho\left(R^{f}\right) \oslash \rho_{\max }\right) \diamond \mu_{R^{f}}\left(v_{\operatorname{var}\left(R^{f}\right)}\right) \\
& =\left(\rho_{0} \oslash \rho_{0}\right) \diamond \mu_{R^{f}}\left(v_{\operatorname{var}\left(R^{f}\right)}\right) \\
& =1 \diamond \mu_{R^{f}}\left(v_{\operatorname{var}\left(R^{f}\right)}\right) \\
& =\mu_{R^{f}}\left(v_{\operatorname{var}\left(R^{f}\right)}\right) .
\end{aligned}
$$

So, the second axiom in Definition 6 is satisfied.

(3) Since T-norm $\triangle$ used in (45) is a priority T-norm, under condition (22) we have (23). So, the third axiom in Definition 6 is satisfied.

(4) By axiom 1 of priority operators in Definition 8, namely $a \diamond b$ increases when $b$ increases, $\forall R^{f} \in C^{f}$, if $\mu_{R^{f}}\left(v_{\operatorname{var}\left(R^{f}\right)}\right) \geqslant \mu_{R^{f}}\left(v_{\operatorname{var}\left(R^{f}\right)}^{\prime}\right)$, then we have

$$
\begin{aligned}
\mu_{R^{f}}^{\rho}\left(v_{\operatorname{var}\left(R^{f}\right)}\right) & =\left(\rho\left(R^{f}\right) \oslash \rho_{\max }\right) \diamond \mu_{R^{f}}\left(v_{\operatorname{var}\left(R^{f}\right)}\right) \\
& \geqslant\left(\rho\left(R^{f}\right) \oslash \rho_{\max }\right) \diamond \mu_{R^{f}}\left(v_{\operatorname{var}\left(R^{f}\right)}^{\prime}\right) \\
& =\mu_{R^{f}}^{\rho}\left(v_{\operatorname{var}\left(R^{f}\right)}^{\prime}\right) .
\end{aligned}
$$

Thus, again noticing the monotonicity of the operator $\triangle$, we know that the fourth axiom in Definition 6 is satisfied.

(5) When there exists a compound label $v_{\operatorname{var}\left(R^{f}\right)}$ such that for each $R^{f} \in C^{f}$

$$
\mu_{R^{f}}\left(v_{\operatorname{var}\left(R^{f}\right)}\right)=1,
$$

by Lemma 3 we have

$$
\mu_{R^{f}}^{\rho}\left(v_{\operatorname{var}\left(R^{f}\right)}\right)=\left(\rho\left(R^{f}\right) \oslash \rho_{\max }\right) \diamond \mu_{R^{f}}\left(v_{\operatorname{var}\left(R^{f}\right)}\right)=\left(\rho\left(R^{f}\right) \oslash \rho_{\max }\right) \diamond 1=1 .
$$

Again according to the fifth axiom of the operator $\triangle$ (i.e., $a \triangle 1=a$ ), $1 \triangle 1=1$. So, the fourth axiom in Definition 6 is satisfied. 
By comparing the PFCSP global satisfaction degree formula (45) with the FCSP global satisfaction degree formula (6), we can see that Theorem 4 actually transforms a PFCSP $\left(X, D, C^{f}, \rho\right)$ into an $\operatorname{FCSP}\left(X, D, C_{\rho}^{f}\right)$ where

$$
C_{\rho}^{f}=\left\{R_{\rho}^{f} \mid \mu_{R_{\rho}^{f}}\left(v_{\operatorname{var}\left(R_{\rho}^{f}\right)}\right)=\mu_{R^{f}}^{\rho}\left(v_{\operatorname{var}\left(R^{f}\right)}\right), R^{f} \in C^{f}\right\} .
$$

After transforming a PFCSP into its equivalent FCSP counterpart, we can employ the methods, developed for solving conventional FCSPs, to solve PFCSPs. Examples of such methods include $[17,19,54]$ and Fuzzy GENET [76,77]. However, currently most FCSP solving algorithms are restricted to min as T-norms, but we use general T-norms. The choice of general T-norms used has a strong influence on the efficiency or applicability of usual constraint solving techniques (such as arc-consistency [55,7,9,8,33]). Accordingly, there should be some differences. The answer to what the differences are would certainly be significant, and so it is worthy of further pursuit. ${ }^{3}$

\section{Validation}

According to the principle of knowledge engineering [70], the validation for a knowledge system should be undertaken both internally and externally. Internally, this section uses a number of examples to check whether our schemes indeed satisfy the axiomatic framework. Externally, this section checks whether our schemes behave consistently with human intuition in several realistic scenarios. Thus, the aims of this section is to obtain some deeper insights into the properties and characteristics of our system.

\subsection{Normalised priorities}

This subsection examines a number of examples to show that if the priorities of constraints in a PFCSP are determined by a voting model (see [67, pp. 204-211]), our scheme indeed satisfies the axioms we propose for PFCSPs.

Voting is a simple way to determine the priority of each constraint. For example, for three constraints $R_{1}^{f}, R_{2}^{f}$ and $R_{3}^{f}$, and ten voters, if $R_{1}^{f}, R_{2}^{f}$ and $R_{3}^{f}$ get 3,4 and 3 votes, respectively, then their priorities are $0.3,0.4$ and 0.3 , respectively. Generally, suppose one voter can vote only for one constraint and the priority of a constraint is defined as the percentage of the voters, who vote for the constraint, to all voters, then we should have

$$
\sum_{R^{f} \in C^{f}} \rho\left(R^{f}\right)=1 .
$$

Namely, we assume that the sum of the priorities of all constraints is equal to 1 . In this case, we say priorities are normalised. Traditionally, (47) is assumed in many important decision-making

\footnotetext{
${ }^{3}$ FCSPs have been studied for many years, but most of their solving algorithms are still restricted to min as T-norms. It seems that designing an efficient solving algorithm for an FCSP with general T-norms is a hard problem. Addressing this problem falls outside the scope of the current paper although it is certainly a subject of further research.
} 
problems $[65,1,29,12]$ where priorities represent the group's or the individual's opinion with regard to the relative importance of each object in a set of objects (e.g., criteria, alternatives). ${ }^{4}$

We believe it is reasonable to determine the priority of each constraint via voting. For example, a family wants to buy a house. Suppose their requirements on the desired house are expressed as fuzzy constraints. Clearly, different family members may have their own preferences on the importance level of constraints. Then, the question is: how to determine the priority of each constraint, by which the preference of each family member is fairly reflected? Voting is one such way; that is, each family member is allowed to vote only for one constraint. Also, voting is a reasonable way for one person to determine the priorities of constraints, which reflects his preference on the importance level of constraints. In fact, he can imagine he has a number of voters (e.g., 100 voters) and one voter is allowed to vote only for one constraint.

In the following, under the normalisation assumption (47), we use a number of numerical examples to check whether our scheme satisfies the five axioms we propose in Definition 6. By Theorem 4 and prioritised fuzzy constraint satisfaction formula (32), under our axiomatic framework, we can use the following formula for calculating the global satisfaction degree of a PFCSP consisting of two constraints $R_{1}^{f}$ and $R_{2}^{f}$ :

$$
\min \left\{\max \left\{1-\frac{\rho\left(R_{1}^{f}\right)}{\rho\left(R_{1}^{f}\right)}, \mu_{R_{1}^{f}}\left(v_{\operatorname{var}\left(R_{1}^{f}\right)}\right)\right\}, \max \left\{1-\frac{\rho\left(R_{2}^{f}\right)}{\rho\left(R_{1}^{f}\right)}, \mu_{R_{2}^{f}}\left(v_{\operatorname{var}\left(R_{2}^{f}\right)}\right)\right\}\right\} .
$$

The following five examples show that our scheme indeed satisfies the five axioms we propose, in Definition 6, for PFCSPs.

Example 7. Suppose $\rho\left(R_{1}^{f}\right)=0.7$ and $\rho\left(R_{2}^{f}\right)=0.3$. For a compound label $v_{X}$, let $\mu_{R_{1}^{f}}\left(v_{\operatorname{var}\left(R_{1}^{f}\right)}\right)=0$ and $\mu_{R_{2}^{f}}\left(v_{\operatorname{var}\left(R_{2}^{f}\right)}\right)=0.3$. Then, by (48) the global satisfaction degree is

$$
\min \left\{\max \left\{1-\frac{0.7}{0.7}, 0\right\}, \max \left\{1-\frac{0.3}{0.7}, 0.3\right\}\right\}=0 .
$$

That is, when the compound label $v_{X}$ violates completely the most important constraint $R_{2}^{f}, v_{X}$ cannot become a solution to the PFCSP consisting of $R_{1}^{f}$ and $R_{2}^{f}$. So, the first axiom is fulfilled in our scheme.

Example 8. Suppose the priorities of the two constraints are the same, i.e., $\rho\left(R_{1}^{f}\right)=\rho\left(R_{2}^{f}\right)=0.5$. For a compound label $v_{X}$, let $\mu_{R_{1}^{f}}\left(v_{\operatorname{var}\left(R_{1}^{f}\right)}\right)=0.8$ and $\mu_{R_{2}^{f}}\left(v_{\operatorname{var}\left(R_{2}^{f}\right)}\right)=0.3$. Then, by (48), the global satisfaction degree is

$$
\min \left\{\max \left\{1-\frac{0.5}{0.5}, 0.8\right\}, \max \left\{1-\frac{0.5}{0.5}, 0.3\right\}\right\}=0.3
$$

\footnotetext{
${ }^{4}$ Notice that the normalisation assumption (47) is allowed in our framework but it is not compulsory for our axiomatic system. In other words, we do not make (47) as an assumption of our axiom system.
} 
Clearly, this result is equal to the following global satisfaction of its non-prioritised counterpart FCSP:

$$
\min \left\{\mu_{R_{1}^{f}}\left(v_{\operatorname{var}\left(R_{1}^{f}\right)}\right), \mu_{R_{2}^{f}}\left(v_{\operatorname{var}\left(R_{2}^{f}\right)}\right)\right\}=\min \{0.8,0.3\}=0.3
$$

That is, when the priorities of constraints $R_{1}^{f}$ and $R_{2}^{f}$ are the same, the PFCSP consisting of $R_{1}^{f}$ and $R_{2}^{f}$ degenerates into a non-prioritised FCSP. So, our scheme obeys the second axiom.

Example 9. Suppose $\rho\left(R_{1}^{f}\right)=0.1$ and $\rho\left(R_{2}^{f}\right)=0.9$. For a compound label $v_{X}$ and another compound label $v_{X}^{\prime}$, let

$$
\begin{aligned}
& \mu_{R_{1}^{f}}\left(v_{\operatorname{var}\left(R_{1}^{f}\right)}\right)=0.6, \quad \mu_{R_{2}^{f}}\left(v_{\operatorname{var}\left(R_{2}^{f}\right)}\right)=0.5+0.35 ; \\
& \mu_{R_{1}^{f}}\left(v_{\operatorname{var}\left(R_{1}^{f}\right)}^{\prime}\right)=0.6+0.35, \mu_{R_{2}^{f}}\left(v_{\operatorname{var}\left(R_{2}^{f}\right)}^{\prime}\right)=0.5 .
\end{aligned}
$$

Then, by (48) we have

$$
\begin{aligned}
& \min \left\{\max \left\{1-\frac{0.1}{0.9}, 0.6\right\}, \max \left\{1-\frac{0.9}{0.9}, 0.5+0.35\right\}\right\}=0.85 \\
& >0.5=\min \left\{\max \left\{1-\frac{0.1}{0.9}, 0.6+0.35\right\}, \max \left\{1-\frac{0.9}{0.9}, 0.5\right\}\right\} .
\end{aligned}
$$

That is, raising the satisfaction degree of $R_{2}^{f}$ with a relatively high priority is more effective than raising the global satisfaction degree of $R_{1}^{f}$ with a relatively low priority. So, the third axiom is satisfied in our scheme.

Example 10. Suppose $\rho\left(R_{1}^{f}\right)=0.1$ and $\rho\left(R_{2}^{f}\right)=0.9$. For a compound label $v_{X}$, let $\mu_{R_{1}^{f}}\left(v_{\operatorname{var}\left(R_{1}^{f}\right)}\right)=0.6$ and $\mu_{R_{2}^{f}}\left(v_{\operatorname{var}\left(R_{2}^{f}\right)}\right)=0.85$; and for a compound label $v_{X}^{\prime}$, let $\mu_{R_{1}^{f}}\left(v_{\operatorname{var}\left(R_{1}^{f}\right)}^{\prime}\right)=0.5$, and $\mu_{R_{2}^{f}}\left(v_{\operatorname{var}\left(R_{2}^{f}\right)}^{\prime}\right)=0.7$. Thus,

$$
\mu_{R_{i}^{f}}\left(v_{\operatorname{var}\left(R_{i}^{f}\right)}\right)>\mu_{R_{i}^{f}}\left(v_{\operatorname{var}\left(R_{i}^{f}\right)}^{\prime}\right), \quad i=1,2 .
$$

Then, by (48) we have

$$
\begin{aligned}
& \min \left\{\max \left\{1-\frac{0.1}{0.9}, 0.6\right\}, \max \left\{1-\frac{0.9}{0.9}, 0.85\right\}\right\}=0.85 \\
& >0.7=\min \left\{\max \left\{1-\frac{0.1}{0.9}, 0.5\right\}, \max \left\{1-\frac{0.9}{0.9}, 0.7\right\}\right\} .
\end{aligned}
$$

That is, when the local satisfaction degree of each constraint for compound label $v_{X}$ is greater than the local satisfaction degree of the constraint for compound label $v_{X}^{\prime}$, the global satisfaction degree for compound label $v_{X}$ is greater than the global satisfaction degree for compound label $v_{X}^{\prime}$. So, our scheme meets the fourth axiom. 
Example 11. Suppose $\rho\left(R_{1}^{f}\right)=0.1$ and $\rho\left(R_{2}^{f}\right)=0.9$. For a compound label $v_{X}$, let $\mu_{R_{1}^{f}}\left(v_{\operatorname{var}\left(R_{1}^{f}\right)}\right)=$ $\mu_{R_{2}^{f}}\left(v_{\operatorname{var}\left(R_{2}^{f}\right)}\right)=1$. Then, by (48) the global satisfaction degree is

$$
\min \left\{\max \left\{1-\frac{0.1}{0.9}, 1\right\}, \max \left\{1-\frac{0.9}{0.9}, 1\right\}\right\}=1
$$

That is, when both constraints $R_{1}^{f}$ and $R_{2}^{f}$ are completely satisfied with a compound label $v_{X}, v_{X}$ is the best solution to the PFCSP consisting of $R_{1}^{f}$ and $R_{2}^{f}$. So, the fifth axiom is satisfied in our scheme.

\subsection{Non-normalised priorities}

This subsection further examines our scheme without normalisation assumption (47). We do not use numerical examples to show our scheme satisfies the proposed axioms again (although it does indeed satisfy the axioms). Instead, we use a real example to show that even if priorities do not obey normalisation assumption (47), our scheme still leads to results that are consistent with human intuition.

Example 12. Suppose an exchange student wants to rent a room from a real estate agent in Hong Kong. He has three constraints:

$R_{1}^{f}$ : He wishes that the room should have some quality but cannot be too expensive.

$R_{2}^{f}$ : Since he is an exchange student for only 1 year, he cannot rent a room for more than one year. Moreover, he likes to move because he wants to make more friends, but he does not want to move too often.

$R_{3}^{f}$ : He would like the room to be within walking distance to the university because this can remove the need for expensive transport journeys and cut down on wasted travelling time.

Precisely, these three constraints are defined in Figs. 1-3, respectively.

Assume he thinks that $R_{2}^{f}$ is the most important one. This is because he likes moving and making friends. And he thinks $R_{1}^{f}$ and $R_{3}^{f}$ are equally important. In this case, the only requirement in our scheme is that $R_{1}^{f}$ and $R_{3}^{f}$ must have the same priority and that this is lower than the one $R_{2}^{f}$. Thus, $\rho\left(R_{1}^{f}\right)=\rho\left(R_{3}^{f}\right)=0.1$ and $\rho\left(R_{2}^{f}\right)=0.2$ is possible and legal in our scheme although they do not satisfy normalisation assumption (47).

Now suppose the real estate agent finds a room for him. The rate and the distance of the room are HK\$2000 per month and 15 min walking to the university, respectively. That is, he gets the perfect price and distance. Unfortunately, the real estate agent tells him that the rental period is at least 24 months. If he cannot stay in Hong Kong for more than one year, he should not take this accommodation since he will waste 12 months rent. Definitely, he should not be satisfied with this room at all. Now, what is the result according to our scheme? Formally, we have

$$
\mu_{R_{1}^{f}}(200)=1, \quad \mu_{R_{2}^{f}}(24)=0, \quad \mu_{R_{3}^{f}}(15)=0.7,
$$




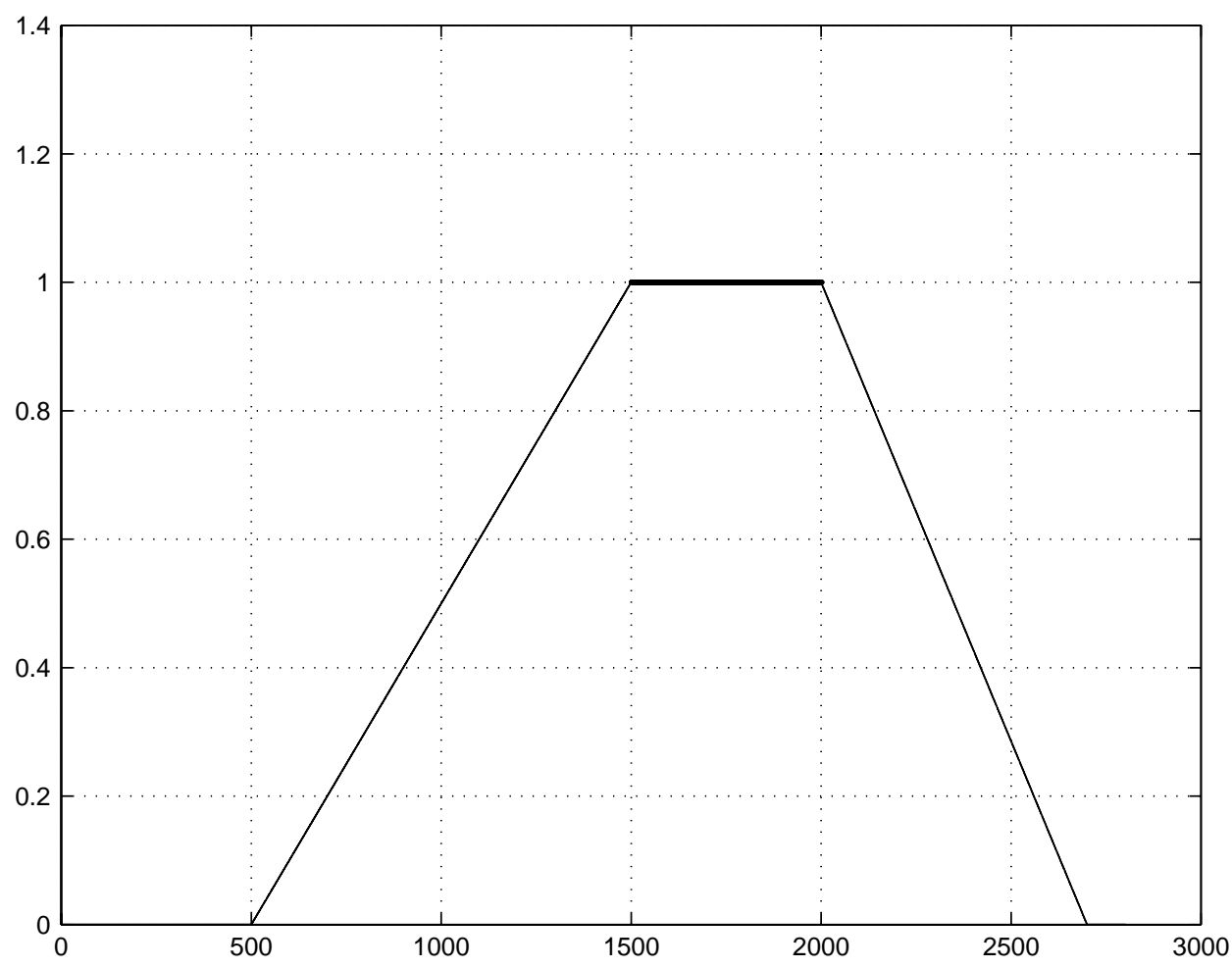

Fig. 1. Fuzzy constraint, $R_{1}^{f}$, on rent (Hong Kong dollars per month).

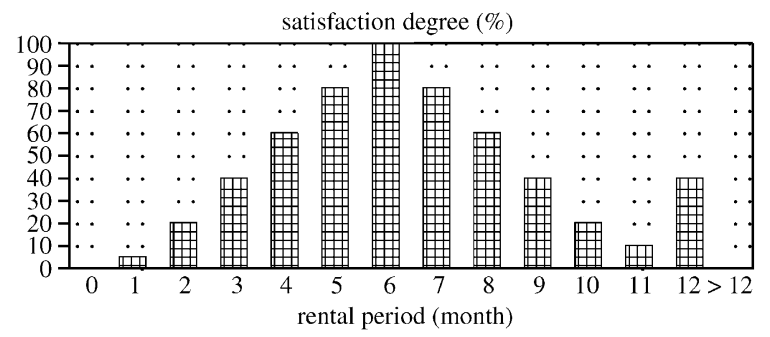

Fig. 2. Fuzz constraint, $R_{2}^{f}$, on rental period.

thus, by (48) in our scheme, the global satisfaction degree is

$$
\min \left\{\max \left\{1-\frac{0.1}{0.2}, 1\right\}, \max \left\{1-\frac{0.2}{0.2}, 0\right\}, \max \left\{1-\frac{0.1}{0.2}, 1\right\}\right\}=0
$$

So, the result, according to our scheme, is still that the student should not be satisfied with the room at all. 


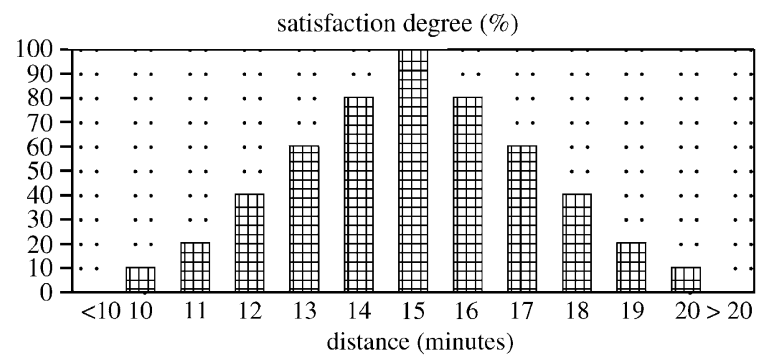

Fig. 3. Fuzzy constraint, $R_{3}^{f}$, on distance.

\subsection{Dynamic situation}

This subsection examines the behaviour of our scheme in dynamic situations.

Example 13. The real world is dynamic and changes over time. Suppose there are three constraints $R_{1}^{f}, R_{2}^{f}$ and $R_{3}^{f}$, and their priorities are changing over time as follows:

$$
(0.2,0.3,0.5) \rightarrow(0.25,0.45,0.3) \rightarrow(0.4,0.3,0.3) \rightarrow \cdots \quad
$$

And suppose the sequence is infinite, and converges to $\left(\frac{1}{3}, \frac{1}{3}, \frac{1}{3}\right)$, namely all priorities converge to the same value $\frac{1}{3}$. In this case, how does the PFCSP global satisfaction degree change? For the three fuzzy constraints $R_{1}^{f}, R_{2}^{f}$ and $R_{3}^{f}$, in our scheme we have

$$
\begin{aligned}
& \quad \lim _{\sum_{1=1}^{3}\left|\rho\left(R_{i}^{f}\right)-\frac{\rho\left(R_{j}^{f}\right)}{\rho_{\max }}\right| \rightarrow 0} \min _{i=1}^{3} \max \left\{1-\rho\left(R_{i}^{f}\right), \mu_{R_{i}^{f}}\left(v_{\operatorname{var}\left(R_{i}^{f}\right)}\right)\right\} \\
& =\min _{i=1}^{3} \max \left\{1-\frac{\frac{1}{3}}{\frac{1}{3}}, \mu_{R_{i}^{f}}\left(v_{\operatorname{var}\left(R_{i}^{f}\right)}\right)\right\} \\
& =\min _{i=1}^{3} \mu_{R_{i}^{f}}\left(v_{\operatorname{var}\left(R_{i}^{f}\right)}\right) .
\end{aligned}
$$

That is, the result is equivalent to that of the non-prioritised counterpart.

This example shows that in such a dynamic situation, our scheme is consistent with human intuition. That is, when all priorities approach, gradually and continually, to the same value, the global satisfaction degree of a PFCSP should also approach, gradually and continually, to the global satisfaction degree of its counterpart FCSP. Our general scheme (45) can also capture this intuition. In fact, we have:

Theorem 5. In a PFCSP $\left(X, D, C^{f}, \rho\right)$, given a compound label $v_{X}$ of all variables in $X$,

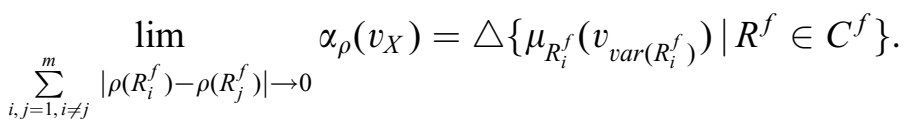




\section{Proof.}

$$
\left.\left.\begin{array}{l}
\sum_{i, j=1, i \neq j}^{m}\left|\rho\left(R_{i}^{f}\right)-\rho\left(R_{j}^{f}\right)\right| \rightarrow 0 \\
=\lim _{\sum_{i, j=1, i \neq j}^{m}\left|\rho\left(R_{i}^{f}\right)-\rho\left(R_{j}^{f}\right)\right| \rightarrow 0} \Delta\left\{\mu_{R^{f}}^{\rho}\left(v_{\operatorname{var}\left(R^{f}\right)}\right) \mid R^{f} \in C^{f}\right\} \\
=\lim _{i, j=1, i \neq j}^{m}\left|\rho\left(R_{i}^{f}\right)-\rho\left(R_{j}^{f}\right)\right| \rightarrow 0 \\
=\triangle\left\{\left(\rho\left(R^{f}\right) \oslash \rho_{\max }\right) \diamond \mu_{R^{f}}\left(v_{\operatorname{var}\left(R^{f}\right)}\right) \mid R^{f} \in C^{f}\right\} \\
=\triangle\left\{\left(\left(\sum_{\sum_{i, j=1, i \neq j}^{m}\left|\rho\left(R_{i}^{f}\right)-\rho\left(R_{j}^{f}\right)\right| \rightarrow 0}\left(\left(\rho\left(R^{f}\right) \oslash \rho_{\max }\right) \diamond \mu_{R^{f}}\left(v_{\operatorname{var}\left(R^{f}\right)}\right)\right) \mid R^{f} \in C^{f}\right\}\right.\right. \\
\lim _{i=1, i \neq j}\left|\rho\left(R_{i}^{f}\right)-\rho\left(R_{j}^{f}\right)\right| \rightarrow 0
\end{array}\right\}\right)
$$

\subsection{Non-monotonicity of decision making}

In many real-world cases, reasoning is non-monotonic [62,53] in nature, i.e., when we obtain some new evidence, the original conclusion we drew needs to be withdrawn. The question then is: how does this work in a PFCSP framework? That is, is it possible to change a previously made decision when we get a new constraint? The answer is affirmative.

First, we give an example using the usual FCSP scheme.

Example 14. A Chinese student wants to pursue Ph.D. degree overseas. He gets two constraints at the beginning:

$R_{1}$ : He wants to go to an English-speaking country. Precisely, his satisfaction degrees (preferences) for different English-speaking countries are as shown in row 2 of Table 1.

$R_{2}$ : The country should not be too cold or too hot. Canada is very cold, while Singapore is very hot. The weather in the USA is similar to China.The UK may be a little colder than China. Precisely, with respect to weather his satisfaction degrees for these countries are as shown in row 3 in Table 1.

According to the two constraints, by using operator min, we obtain his global satisfaction degree for the countries as shown in row 4 of Table 1. At the moment, the USA is the best choice for him according to row 4 of Table 1 . Thus, he begins to prepare to go there. Then, he finds that he 
Table 1

The country selecting problem based on a FCSP

\begin{tabular}{llllll}
\hline & USA & Canada & UK & Australia & Singapore \\
\hline$R_{1}$ & $100 \%$ & $80 \%$ & $90 \%$ & $70 \%$ & $50 \%$ \\
$R_{2}$ & $100 \%$ & $40 \%$ & $80 \%$ & $60 \%$ & $40 \%$ \\
$R_{1} \& R_{2}$ & $100 \%$ & $40 \%$ & $80 \%$ & $60 \%$ & $40 \%$ \\
$R_{3}$ & $40 \%$ & $60 \%$ & $80 \%$ & $60 \%$ & $60 \%$ \\
$R_{1} \& R_{2} \& R_{3}$ & $40 \%$ & $40 \%$ & $80 \%$ & $60 \%$ & $40 \%$ \\
\hline
\end{tabular}

Table 2

The country selecting problem based on our scheme for PFCSPs

\begin{tabular}{llllll}
\hline & USA & Canada & UK & Australia & Singapore \\
\hline$R_{1}$ & $100 \%$ & $80 \%$ & $90 \%$ & $70 \%$ & $50 \%$ \\
$R_{2}$ & $100 \%$ & $50 \%$ & $80 \%$ & $60 \%$ & $50 \%$ \\
\hline$R_{1} \& R_{2}$ & $100 \%$ & $50 \%$ & $80 \%$ & $60 \%$ & $50 \%$ \\
$R_{3}$ & $40 \%$ & $60 \%$ & $80 \%$ & $60 \%$ & $60 \%$ \\
$R_{1} \& R_{2} \& R_{3}$ & $40 \%$ & $50 \%$ & $80 \%$ & $60 \%$ & $50 \%$ \\
\hline
\end{tabular}

must take both TOEFL and GRE exams. However, taking both the tests is too demanding for him because he wants to go as soon as possible. Thus, he gets the third constraint:

$R_{3}$ : Minimise the effort on English. Precisely, with respect to English tests, his satisfaction degrees for the countries are as shown in row 5 of Table 1.

As a result, the UK instead of the USA is the best choice for him according to row 6 of Table 1, which is obtained by using the $\min$ operator.

The above example shows that the framework of FCSPs can capture non-monotonicity in decision making. In the following example we can see that our scheme can also capture non-monotonicity of this form.

Example 15. In Example 14, suppose the student thinks that the first constraint is the most important, the second one is the least important, and the importance of the third constraint lies in between. Thus, in our framework he can assign the three constraints the priorities of 8,6 and 4 , respectively. By prioritised fuzzy constraint satisfaction degree formula (32), we obtain the local satisfaction degrees of these prioritised fuzzy constraints as shown in rows 2, 3 and 5 of Table 2. Like Example 14, at the beginning he just takes the first two constraints into account; by using operator min, we obtain his global satisfaction degrees for the different countries as shown in row 4 of Table 2 (so the USA is the best choice for him at the moment). Further he puts the third constraint into consideration; 
by using operator min, his global satisfaction degrees for different countries change to the ones as shown in row 6 of Table 2 (so the UK instead of the USA is the best choice for him now).

The following theorem gives a sufficient condition for how adding a new constraint would change the relative ordering of the global satisfaction degrees of compound labels.

Theorem 6. For a PFCSP, suppose the global satisfaction degree for compound label $v_{X}$ is $\alpha_{\rho}^{\text {old }}\left(v_{X}\right)$, and the global satisfaction degree for compound label $v_{X}^{\prime}$ is $\alpha_{\rho}^{\text {old }}\left(v_{X}^{\prime}\right)$. For a new constraint $R$ which is not in the PFCSP, if

$$
\alpha_{\rho}^{\text {old }}\left(v_{X}^{\prime}\right) \triangle \mu_{R}\left(v_{v a r}^{\prime}(R)\right) \geqslant \min \left\{\alpha_{\rho}^{\text {old }}\left(v_{X}\right), \mu_{R}\left(v_{v a r}(R)\right)\right\},
$$

where $\triangle$ is a priority T-norm that is used to calculate the global satisfaction degree by aggregating the local satisfaction degrees of constraints, then for the new PFCSP which is obtained via adding constraint $R$ into the old PFCSP, the ordering of the global satisfaction degrees for $v_{X}$ and $v_{X}^{\prime}$ is

$$
\alpha_{\rho}^{\text {new }}\left(v_{X}\right) \leqslant \alpha_{\rho}^{\text {new }}\left(v_{X}^{\prime}\right) .
$$

\section{Proof.}

$$
\begin{aligned}
\alpha_{\rho}^{\text {new }}\left(v_{X}\right) & =\alpha_{\rho}^{\text {old }}\left(v_{X}\right) \triangle \mu_{R}\left(v_{\operatorname{var}(R)}\right) \quad(\text { by Theorem } 4) \\
& \leqslant \min \left\{\alpha_{\rho}^{\text {old }}\left(v_{X}\right), \mu_{R}\left(v_{\operatorname{var}(R)}\right)\right\} \quad(\text { by Lemma } 1) \\
& \leqslant \alpha_{\rho}^{\text {old }}\left(v_{X}^{\prime}\right) \triangle \mu_{R}\left(v_{\operatorname{var}(R)}^{\prime}\right) \quad(\text { by }(50)) \\
& =\alpha_{\rho}^{\text {new }}\left(v_{X}^{\prime}\right) . \quad(\text { by Theorem } 4)
\end{aligned}
$$

This theorem shows that no matter what the ordering is on the old global satisfaction degrees of two compound labels, as long as inequality (50) holds, the ordering of the new global satisfaction degrees of the two compound labels is (51). So, if before adding new constraint $R$ we have

$$
\alpha_{\rho}^{\text {old }}\left(v_{X}\right) \geqslant \alpha_{\rho}^{\text {old }}\left(v_{X}^{\prime}\right),
$$

as long as inequality (50) holds, the ordering (52) changes to (51).

\section{Posterioritised FCSPs}

Traditionally, the concept of weight is used to indicate the importance level of an object among some objects [3,36,28,13-15] and, so like the concept of priority, it can also be used to indicate the importance level of a constraint among some constraints. Given this, an obvious question to be asked is: what is the difference between them? In this section, we clarify the relationship between the schemes for prioritised FCSPs and the schemes for weighted FCSPs. That is, in contrast to prioritised FCSPs, weighted FCSPs can be called posterioritised FCSPs since the solutions of weighted FCSPs mainly depend on constraints with posteriority (relatively low importance level), whereas the solutions of prioritised FCSP schemes mainly depend on constraints with priority (relatively high 
importance level). So, in this sense, a scheme for weighted FCSPs is the dual of the scheme of PFCSPs.

First, we recall a number of relevant concepts and notations. T-norm and T-conorm are special cases of the concept of uninorm operators $[30,78-80,38,43]$ defined as follows.

Definition 10. A binary operator $\oplus^{(\tau)}:[0,1] \times[0,1] \rightarrow[0,1]$ is a uninorm if it is increasing, associative and commutative and there exists $\tau \in[0,1]$ such that

$$
\forall a \in[0,1], a \oplus^{(\tau)} \tau=a
$$

Here $\tau$ is called the unit element of the uninorm.

Clearly, when $\tau=1$, a uninorm is a T-norm; when $\tau=0$, a uninorm is a T-conorm. For $\tau \in(0,1)$, Klement et al. [38] showed that the following parallel combination formula in the PROSPECTOR uncertain reasoning model $[26,82,47,49,50]$ is a uninorm operator:

$$
a_{1} \oplus^{(\tau)} a_{2}=\frac{(1-\tau) a_{1} a_{2}}{(1-\tau) a_{1} a_{2}+\tau\left(1-a_{1}\right)\left(1-a_{2}\right)},
$$

where $\tau \in(0,1)$ is the unit element.

Further, Yager and Rybalov [79] introduced the weighted uninorm aggregation:

Definition 11. A weighted uninorm aggregation (WUA) of dimension $n$ is a mapping $F_{W U A}:[0,1]^{n} \times$ $[0,1]^{n} \rightarrow[0,1]$, defined as

$$
F_{W U A}(\vec{a}, \vec{w})=\left(w_{1} \diamond^{(\tau)} a_{1}\right) \oplus^{(\tau)} \cdots \oplus^{(\tau)}\left(w_{n} \diamond^{(\tau)} a_{n}\right),
$$

where:

(1) $\vec{a}=\left(a_{1}, \ldots, a_{n}\right), \vec{w}=\left(w_{1}, \ldots, w_{n}\right)$ such that $w_{i}$ is the weight of $a_{i}(0 \leqslant i \leqslant n)$;

(2) $\oplus^{(\tau)}$ is a uninorm operator with unit element $\tau \in[0,1]$;

(3) operator $\diamond^{(\tau)}:[0,1] \times[0,1] \rightarrow[0,1]$, called a weight operator with respect to uninorm $\oplus^{(\tau)}$, satisfies:

(i) $\forall a_{1}, a_{2}, a_{2}^{\prime} \in[0,1], a_{2} \leqslant a_{2}^{\prime} \Rightarrow a_{1} \diamond^{(\tau)} a_{2} \leqslant a_{1} \diamond^{(\tau)} a_{2}^{\prime}$,

(ii) $\forall a_{1}, a_{1}^{\prime} \in[0,1], a_{2} \in[\tau, 1], a_{1} \leqslant a_{1}^{\prime} \Rightarrow a_{1} \diamond^{(\tau)} a_{2} \leqslant a_{1}^{\prime} \diamond^{(\tau)} a_{2}$,

(iii) $\forall a_{1}, a_{1}^{\prime} \in[0,1], a_{2} \in[0, \tau], a_{1} \leqslant a_{1}^{\prime} \Rightarrow a_{1} \diamond^{(\tau)} a_{2} \geqslant a_{1}^{\prime} \diamond^{(\tau)} a_{2}$

(iv) $\forall a \in[0,1], 1 \diamond^{(\tau)} a=a$, and

(v) $\forall a \in[0,1], 0 \diamond^{(\tau)} a=\tau$.

In the following, based on the idea behind the weighted uninorm aggregation defined above, and the idea behind the relatively weighted logic [48], we introduce the concept of weighted fuzzy constraint problems. Notice that we restrict unit element $\tau=0$ of uninorm operators. In other words, we use a special case of uninorm operators: T-norms. The reason for this is simply for the sake of compatibility between WFCSPs and PFCSPs. That is, in WFCSPs T-norms should be employed in 
aggregating the local satisfaction degrees of weighted fuzzy constraints since in PFCSPs a kind of T-norms are employed in aggregating local satisfaction degrees of prioritised fuzzy constraints.

Definition 12. A weighted fuzzy constraint satisfaction problem (WFCSP) is a 4-tuple $\left(X, D, C^{f}\right.$, $\omega)$, where $\left(X, D, C^{f}\right)$ is an FCSP and is called the counterpart FCSP of the WFCSP, and $\omega: C^{f} \rightarrow$ $[0, \infty)$ is a weight function. In $\operatorname{WFCSP}\left(X, D, C^{f}, \omega\right)$, given a compound label $v_{X}$ of all variables in $X$, the global satisfaction degree is given by

$$
\alpha_{\omega}\left(v_{X}\right)=\triangle\left\{\left(\omega\left(R^{f}\right) \oslash \omega_{\max }\right) \diamond \mu_{R^{f}}\left(v_{\operatorname{var}\left(R^{f}\right)}\right) \mid R^{f} \in C^{f}\right\}
$$

where $\oslash$ is a general division operator, $\omega_{\max }=\max \left\{\omega\left(R^{f}\right) \mid R^{f} \in C^{f}\right\}, \diamond$ is a weight operator with respect to T-norm $\triangle$. A solution to $\operatorname{WFCSP}\left(X, D, C^{f}, \omega\right)$ is a compound label $v_{X}$ of all variables in $X$ such that

$$
\alpha_{\omega}\left(v_{X}\right) \geqslant \alpha_{0}
$$

where $\alpha_{0} \in[0,1]$, called the WFCSP's solution threshold, is predetermined.

Before discussing the relationship between WFCSPs and PFCSPs, we give one property about priority operators.

Theorem 7. Suppose operator $\diamond$ satisfies axioms 2 and 3 of priority operators, which are listed in Definition 8. Then

$$
a_{1} \diamond a_{2} \geqslant a_{2}
$$

Proof. By axioms 2 and 3 of priority operators (listed in Definition 8), we have

$$
a_{1} \diamond a_{2} \geqslant 1 \diamond a_{2}=a_{2}
$$

The following theorem identifies the difference between priority operators and weight operators.

Theorem 8. $\forall a_{1}, a_{2} \in[0,1]$,

$$
a_{1} \diamond a_{2} \leqslant a_{2} \leqslant a_{1} \diamond a_{2}
$$

Proof. By axioms 2 and 4 of weight operators (which are listed in Definition 11), we have

$$
0 \diamond a_{2} \leqslant a_{1} \diamond a_{2} \leqslant 1 \diamond a_{2}=a_{2} .
$$

And noticing Theorem 7, we have (59).

The above theorem means that the prioritised value is not smaller than the original value, whereas the weighted value is not larger than the original value. This implies that the local satisfaction degree of a constraint with priority is able to exert a non-negative effect upon the global satisfaction degree, 
whereas the local satisfaction degree of a constraint with weight is able to take a non-positive effect upon the global satisfaction degree. In fact, we have the following theorem:

Theorem 9. For a PFCSP $\left(X_{1}, D_{1}, C_{1}^{f}, \rho\right)$ and a $\operatorname{WFCSP}\left(X_{2}, D_{2}, C_{2}^{f}, \omega\right)$, suppose $X_{1}=X_{2}, D_{1}=D_{2}$, $C_{1}^{f}=C_{2}^{f}, \rho=\omega$, and the T-norm operator in their global satisfaction degree formulae is min. If for a compound label $v_{X}$,

$$
\begin{aligned}
& \alpha_{\rho}\left(v_{X}\right)=\left(\rho\left(R_{i}^{f}\right) \oslash \rho_{\max }\right) \diamond \mu_{R_{i}^{f}}\left(v_{\operatorname{var}\left(R_{i}^{f}\right)}\right), \\
& \alpha_{\omega}\left(v_{X}\right)=\left(\omega\left(R_{j}^{f}\right) \oslash \omega_{\max }\right) \diamond \mu_{R_{j}^{f}}\left(v_{\operatorname{var}\left(R_{j}^{f}\right)}\right),
\end{aligned}
$$

then

$$
\omega\left(R_{i}^{f}\right) \geqslant \omega\left(R_{j}^{f}\right)
$$

Proof. First, we notice that operator min is used for the global satisfaction degree in the PFCSP and the WFCSP, and thus we have

$$
\begin{aligned}
& \left(\rho\left(R_{i}^{f}\right) \oslash \omega_{\max }\right) \diamond \mu_{R_{i}^{f}}\left(v_{\operatorname{var}\left(R_{i}^{f}\right)}\right) \leqslant\left(\rho\left(R_{j}^{f}\right) \oslash \omega_{\max }\right) \diamond \mu_{R_{j}^{f}}\left(v_{\operatorname{var}\left(R_{j}^{f}\right)}\right), \\
& \left(\omega\left(R_{i}^{f}\right) \oslash \rho_{\max }\right) \diamond \mu_{R_{i}^{f}}\left(v_{\operatorname{var}\left(R_{i}^{f}\right)}\right) \geqslant\left(\omega\left(R_{j}^{f}\right) \oslash \omega_{\max }\right) \diamond \mu_{R_{j}^{f}}\left(v_{\operatorname{var}\left(R_{j}^{f}\right)}\right) .
\end{aligned}
$$

Then, we have the following two cases:

(1) When $\mu_{R_{i}^{f}}\left(v_{\operatorname{var}\left(R_{i}^{f}\right)}\right) \geqslant \mu_{R_{j}^{f}}\left(v_{\operatorname{var}\left(R_{j}^{f}\right)}\right)$, since priority operator $\diamond$ is decreasing for its first operand and increasing for its second operand and the general division operator $\oslash$ is increasing for its first operand, we must have

$$
\rho\left(R_{i}^{f}\right) \geqslant \rho\left(R_{j}^{f}\right)
$$

otherwise (63) does not hold. And noticing $\rho=\omega$, we have (62).

(2) When $\mu_{R_{i}^{f}}\left(v_{\operatorname{var}\left(R_{i}^{f}\right)}\right) \leqslant \mu_{R_{j}^{f}}\left(v_{\operatorname{var}\left(R_{j}^{f}\right)}\right)$, since $\diamond$ is increasing for its two operands and the general division operator $\oslash$ is increasing for its first operand, we must have (62) otherwise (64) does not hold.

The theorem below reveals the difference relationship between PFCSPs and WFCSPs. That is, in a prioritised FCSP scheme, the global satisfaction degree of all prioritised constraints depends mainly on the local satisfaction degree of a constraint with relatively high importance, whereas in a weighted FCSP scheme the global satisfaction degree of all weighted constraints depends mainly on the local satisfaction degree of a constraint with relatively low importance. The following is such an example.

Example 16. Suppose there are four fuzzy constraints $R_{1}, R_{2}, R_{3}$ and $R_{4}$. Their importance levels are as shown in the second column of Table 3. For a compound label $v_{X}$, their local satisfaction degrees, weighted local satisfaction degrees and prioritised local satisfaction degrees are shown in the third, fourth and fifth columns of Table 3. By using operator min, we find the WFCSP global 
Table 3

Example for the comparison of PFCSPs and WFCSPs ( $\mathrm{SD}=$ satisfaction degree)

\begin{tabular}{lllll}
\hline & Importance & SD & Weighted SD & Prioritised SD \\
\hline$R_{1}$ & $30 \%$ & 0.8 & $\frac{3}{9} \times 0.8$ & $\max \left\{1-\frac{3}{9}, 0.8\right\}$ \\
$R_{2}$ & $70 \%$ & 0.3 & $\frac{7}{9} \times 0.3$ & $\max \left\{1-\frac{7}{9}, 0.3\right\}$ \\
$R_{3}$ & $50 \%$ & 0.5 & $\frac{5}{9} \times 0.4$ & $\max \left\{1-\frac{5}{9}, 0.4\right\}$ \\
$R_{4}$ & $90 \%$ & 0.7 & $\frac{9}{9} \times 0.7$ & $\max \left\{1-\frac{9}{9}, 0.7\right\}$ \\
\hline
\end{tabular}

satisfaction degree is $\frac{5}{9} \times 0.4$, i.e., the local satisfaction degree of weighted $R_{3}$; by using operator $\mathrm{min}$, we find the PFCSP global satisfaction degree is $\max \left\{1-\frac{7}{9}, 0.3\right\}$, i.e., the local satisfaction degree of prioritised $R_{2}$. According to Table $3, R_{2}$ is prior to $R_{3}$. That is, in a prioritised FCSP scheme the global satisfaction degree of all prioritised constraints depends mainly on the local satisfaction degree of a constraint with relatively high importance, whereas in a weighted FCSP scheme the global satisfaction degree of all weighted constraints depends mainly on the local satisfaction degree of a constraint with relatively low importance.

From this, it can be seen that so-called weighted schemes are the dual of prioritised ones. So, in order to capture this characteristic and distinguish it from the prioritised scheme, it would be better if we give this kind of scheme a new name. We propose posterioritised FCSP schemes since the global satisfaction degree of this kind of schemes mainly depends on constraints with relatively low priority (or say relatively high posteriority). Correspondingly, weight operators with respect to T-norms can be called posteriority operators.

\section{Related work}

Besides the relationship between prioritised FCSPs and weighted FCSPs as discussed above, this section undertakes a detailed comparison of our work with that of Dubois et al. [19,20,23]. A summary of the comparison is first given in Table 4, and then its details are discussed point by point in the subsections of the section.

Table 4

Comparison of our work with the work of Dubois, Fargier and Prade (DFP)

\begin{tabular}{llll}
\hline & & The DFP work & Our work \\
\hline Axioms & $\begin{array}{l}\text { Local } \\
\text { Global }\end{array}$ & Yes, but redundant & Yes \\
& No & Yes \\
Scheme & $\begin{array}{l}\text { Axiom } \\
\text { Construction }\end{array}$ & $\begin{array}{l}\text { Does not satisfy all in any case } \\
\text { Yes }\end{array}$ & $\begin{array}{l}\text { Satisfy all } \\
\text { Some new }\end{array}$ \\
Application & User-friendliness & $\begin{array}{l}\text { Better when no hard constraint, } \\
\text { but may behave undesiredly }\end{array}$ & Better in dynamic, \\
& Non-monotonicity & Yes & Yes \\
& & & Yize problem
\end{tabular}


Before giving the detail, we have to point out that the following discussion is carried out under the assumption that in both our work and the work of Dubois et al. the priority of a constraint is understood as the importance level of the constraint. In their work, sometimes the priority of a constraint is understood as a degree to which the constraint is necessarily satisfied (see [23]: the second paragraph of its Section 3.2 in p. 49); sometimes it is understood as the importance level of the constraint (see [23]: its second paragraph in p. 50); sometimes it is viewed as the maximum degree of acceptability of a solution that violates this constraint (see [66]: the last second sentence of its abstract in p. 361). However, among the various papers related to the concept of priority, most authors understand this concept as the importance level of an object concerned since this understanding is in accord with the meaning of word priority in English dictionaries (e.g., [58]). So, it is reasonable to assume that in both systems a constraint's priority represents its importance level. Of course, if in their work the priority of each constraint is interpreted only as the necessity of the constraint's satisfaction, we can simply say that our system is fundamentally different from theirs since in our system the priority only means the importance level. ${ }^{5}$

\subsection{Axioms}

This subsection discusses the comparison of the axiom aspect in Table 4.

First, comparatively few axioms about the global properties of PFCSPs are revealed in the work of Dubois et al. [19,20,23]. The axioms they reveal are mainly about the local properties of PFCSPs, i.e., the axioms of priority operators.

Second, although the concept of priority operators is introduced by Dubois et al. [19,20,23], one of their axioms for priority operators is redundant. Precisely, except for the four axioms we list in Definition 8, they also regard (58) as an axiom that priority operators should satisfy. However, by the proof of Theorem 7, (58) can be derived from two of the four axioms (axioms 2 and 3) listed in Definition 8.

\subsection{Scheme}

This subsection discusses the comparison of the scheme aspect in Table 4.

\subsubsection{Scheme vs. axioms}

The following theorem tells us that the DFP scheme does not satisfy all axioms in Definition 6 in any case.

Theorem 10. The DFP scheme satisfy axioms 1 and 2 in Definition 6 if and only if the most important constraint is assigned priority 1.

Proof. First, the DFP scheme satisfies axioms 1 and 2 if the most important constraint is assigned priority 1. In fact, according to the corresponding formula in the DFP scheme, the complete violation of a constraint with priority 1 yields a completely unacceptable compound label. So, the first axiom

\footnotetext{
${ }^{5}$ Notice that our concept of priority does not necessarily represent voting rate. Voting is just a simple way to determine priorities of constraints (see Section 5.1).
} 
is satisfied. If each constraint has the same priority, this priority is the highest priority and is therefore equal to 1 . Such a PFCSP therefore has all constraints being prioritised by 1 , and therefore degenerates into its non-prioritised counterpart FCSP. So, the second axiom is satisfied.

Second, the DFP scheme cannot satisfy axioms 1 and 2 if the most important constraint is not assigned priority 1 . In fact, we have the following examples:

(1) Under the same assumption of Example 7, that is, $\rho\left(R_{1}^{f}\right)=0.7, \rho\left(R_{2}^{f}\right)=0.3, \mu_{R_{1}^{f}}\left(v_{\operatorname{var}\left(R_{1}^{f}\right)}\right)=0$ and $\mu_{R_{2}^{f}}\left(v_{\operatorname{var}\left(R_{2}^{f}\right)}\right)=0.3$, by the PFCSP global satisfaction degree formula in the DFP scheme

$$
\min \left\{\max \left\{1-\rho\left(R_{1}^{f}\right), \mu_{R_{1}^{f}}\left(v_{\operatorname{var}\left(R_{1}^{f}\right)}\right)\right\}, \max \left\{1-\rho\left(R_{2}^{f}\right), \mu_{R_{2}^{f}}\left(v_{\operatorname{var}\left(R_{2}^{f}\right)}\right)\right\}\right\},
$$

the global satisfaction degree is

$$
\min \{\max \{1-0.7,0\}, \max \{1-0.3,0.3\}\}=0.3 .
$$

This means that the compound label can still be accepted, to some extent, as a solution even if the most important constraint $R_{1}^{f}$ is completely violated. So, the first axiom proposed in Definition 6 is not satisfied in the DFP scheme.

(2) Under the same assumption of Example 8, that is, $\rho\left(R_{1}^{f}\right)=\rho\left(R_{2}^{f}\right)=0.5, \mu_{R_{1}^{f}}\left(v_{\operatorname{var}\left(R_{1}^{f}\right)}\right)=0.8$ and $\mu_{R_{2}^{f}}\left(v_{\operatorname{var}\left(R_{2}^{f}\right)}\right)=0.3$, by (65) in the DFP scheme the global satisfaction degree is

$$
\min \{\max \{1-0.5,0.8\}, \max \{1-0.5,0.3\}\}=0.5 \text {. }
$$

Clearly, this result is not equal to the following global satisfaction of its non-prioritised counterpart FCSP:

$$
\min \left\{\mu_{R_{1}^{f}}\left(v_{\operatorname{var}\left(R_{1}^{f}\right)}\right), \mu_{R_{2}^{f}}\left(v_{\operatorname{var}\left(R_{2}^{f}\right)}\right)\right\}=\min \{0.8,0.3\}=0.3 .
$$

That is, a PFCSP defined in the sense of the DFP scheme cannot degenerate into its non-prioritised counterpart FCSP when all the constraints' priorities are the same. So, the second axiom in Definition 6 does not hold in the DFP scheme.

(3) Notice that in the above two examples, the normalisation assumption (47) is made in determining priorities. The following example shows that in the case that the most important constraint is not assigned priority 1, even if (47) is not assumed, the first two axioms in Definition 6 cannot be satisfied in the DFP scheme, either.

If $R_{1}^{f}, R_{2}^{f}$ and $R_{3}^{f}$ get 3,4 and 3 votes, respectively, the only requirement in the DFP scheme is that $R_{1}^{f}$ and $R_{3}^{f}$ must have the same priority and that this is lower than the one of $R_{2}^{f}$. Thus, $\rho\left(R_{1}^{f}\right)=\rho\left(R_{3}^{f}\right)=0.1$ and $\rho\left(R_{2}^{f}\right)=0.2$ are possible and legal in the DFP scheme. Now let $\mu_{R_{1}^{f}}\left(v_{\operatorname{var}\left(R_{1}^{f}\right)}\right)$ $=0.1, \mu_{R_{3}^{f}}\left(v_{\operatorname{var}\left(R_{3}^{f}\right)}\right)=0.7$, and $\mu_{R_{2}^{f}}\left(v_{\operatorname{var}\left(R_{2}^{f}\right)}\right)=0$. Note that the most important constraint $R_{2}^{f}$ is absolutely unsatisfied with the compound label. Now by the corresponding formula in the DFP scheme, the global satisfaction degree is

$$
\min \{\max \{1-0.1,0.1\}, \max \{1-0.2,0\}, \max \{1-0.1,0.7\}\}=0.8 \neq 0 .
$$

That is, even if a compound label violates completely the most important constraint $R_{2}^{f}$, the global satisfaction degree for the compound label as a solution is $80 \%$ instead of 0 . So, the first axiom in 
Definition 6 is not satisfied in the DFP scheme in this case. Now if $R_{2}^{f}$ is removed for some reason, then the global satisfaction degree becomes

$$
\min \{\max \{1-0.1,0.1\}, \max \{1-0.1,0.7\}\}=0.9 \neq \min \{0.1,0.7\} .
$$

That is, even if two constraints have the same priorities, the PFCSP does not degenerate into its FCSP counterpart. So, the second axiom in Definition 6 is not satisfied in the DFP scheme in this situation.

In the DFP scheme, there is no requirement that the most important constraint must be assigned priority 1. In other words, the DFP scheme suggests that sometimes as long as some fuzzy constraints can be satisfied with a compound label to some extent, even if one of the most important constraints is completely broken, people could still accept, more or less, the compound label as a solution. It seems that this could be regarded as an advantage of the DFP scheme since this feature allows users to express the fact that no constraint should be considered as being hard. However, it may lead undesirable results in some practical applications. In the following, we give two such examples.

Example 17. Suppose the priorities of $R_{1}^{f}, R_{2}^{f}$ and $R_{3}^{f}$ are $\rho\left(R_{1}^{f}\right)=\rho\left(R_{3}^{f}\right)=0.1$ and $\rho\left(R_{2}^{f}\right)=0.2$. And let $\mu_{R_{1}^{f}}\left(v_{\operatorname{var}\left(R_{1}^{f}\right)}\right)=\mu_{R_{3}^{f}}\left(v_{\operatorname{var}\left(R_{3}^{f}\right)}\right)=\mu_{R_{2}^{f}}\left(v_{\operatorname{var}\left(R_{2}^{f}\right)}\right)=0$. Then, by the corresponding formula in the DFP scheme, the global satisfaction degree is

$$
\min \{\max \{1-0.1,0\}, \max \{1-0.2,0\}, \max \{1-0.1,0\}\}=80 \% .
$$

That is, even if a compound label violates completely all constraints, the global satisfaction degree of the compound label as a solution is very high (80\%). Clearly, this result is not always desirable in practical application.

Example 18. In the room renting problem described in Example 12, the result of our scheme is consistent with human intuition, namely the student should not be satisfied with this room at all. However, according to the DFP scheme, the student could be highly satisfied with the room. In fact, in the DFP scheme, the global satisfaction degree is

$$
\min \{\max \{1-0.1,1\}, \max \{1-0.2,0\}, \max \{1-0.1,1\}\}=80 \% .
$$

This result means that the student would like the room to the extent of $80 \%$ without regard to the extra 12 months of rent that has to be unnecessarily paid. Clearly, such a result is undesirable in this practical application.

However, in the above two examples, if the most important constraint $R_{2}$ is assigned priority 1 , such undesirable results will not occur in the practical application. Of course, in a certain circumstance, if the user desires such special results, that is another story. Here by using the above two examples, we do not aim at showing our schemes' superiority over the DFP scheme. What we want to reveal is that the DFP scheme could lead to such special results. So, when applying the DFP scheme to practical problems, the users need to think about whether such special results are desired or not, and accordingly make a decision about whether they need to assign priority 1 to the most 
important constraint. ${ }^{6}$ If one does not want to do so and at the same time does not want such special results either, a safe way is to employ our schemes.

\subsubsection{Scheme construction}

Basically, we obtain some new results for constructing the PFCSP schemes. First, although Dubois and Prade give the axioms for priority operators [23], they just use

$$
\mu_{R^{f}}^{\rho}\left(v_{\operatorname{var}\left(R^{f}\right)}\right)=\rho\left(R^{f}\right) \diamond \mu_{R^{f}}\left(v_{\operatorname{var}\left(R^{f}\right)}\right),
$$

instead of (28), to calculate the local satisfaction degree of a prioritised constraint. Moreover, they just give a special priority operator:

$$
a_{1} \diamond a_{2}=\max \left\{1-a_{1}, a_{2}\right\} .
$$

In contrast, this paper presents five methods for constructing priority operators. The first method is given in Theorem 1. Actually, it is a generalisation of formula (67). That is, operator max in (67) is generalised to a T-conorm. In the community of fuzzy mathematics, many T-conorms have already constructed, and thus we can get many priority operators from these existing T-conorms. Theorem 2 in this paper gives another four methods to construct priority operators. By means of these four methods, we construct four priority operators (37)-(40). Theorem 3 gives the fifth method.

Second, we identify a subclass of T-norms, called priority T-norms, as the aggregation operator to calculate the PFCSP global satisfaction degree by aggregating the local satisfaction degree of each prioritised fuzzy constraint in our framework. These kinds of operators include the Zadeh T-norm operator $\min$ as a special case, and at the same time, like operator min, only when the smaller operand is raised, is the result of aggregation using such an operator raised. However, they only employ a special priority operator: min.

\subsection{Application}

This subsection discusses the comparison of the application aspect in Table 4.

\subsubsection{User-friendliness}

As we have discussed in Section 7.2.1, when the DFP scheme is applied into practice, it is better than our schemes if no hard constraints are required, but in this case the users need to make sure whether some special results are in need. As shown in the proof of Theorem 10, the DFP scheme would not lead to undesirable results in the case where the most important constraint is assigned priority 1 . Nevertheless, from a practical view, it may be uncomfortable for a user to often be forced to assign 1 to every constraint with the highest priority. In fact, it is difficult for a user to know in advance which constraint(s) should have the highest priority, especially in the case where there are large numbers of constraints. For example, if there are 10,000 constraints, it is difficult for a user to decide which one has the absolute priority 1 by comparing them in a pairwise manner. In other words, in order for the DFP scheme to work properly, the problem now becomes how to specify the priorities of the constraints in such a way that the highest priority constraint will always have

\footnotetext{
${ }^{6}$ Note it is impossible to assign priority 1 to the constraint that has the highest priority under assumption (47) of a voting model unless all other constraints have priority 0 .
} 
an absolute priority of 1 . Unfortunately, the DFP scheme is silent on how to achieve this. Such a tedious task should not be left entirely to users, as required by the DFP scheme. This is because such inconvenience in using a system may result in the loss of potential users.

Our framework does not have this problem. Indeed, one of the contributions of our framework is the relaxation of the scale $[0,1]$ to $[0, \infty)$ for priorities, ${ }^{7}$ and the provision of the $\oslash$ operator (axiomatised in Definition 8). This $\oslash$ operator gives one way of automatically transforming a PFCSP with arbitrary priorities into one in which the constraints of highest priority have absolute priority 1. In fact, given a PFCSP $\left(X, D, C^{f}, \rho\right)$, for the priority $\rho\left(R^{f}\right)$ of constraint $R^{f}$, we can define its relative priority $\rho^{\prime}\left(R^{f}\right)$ by

$$
\rho^{\prime}\left(R^{f}\right)=\rho\left(R^{f}\right) \oslash \rho_{\max },
$$

where $\oslash$ is a general division operator. Thus, if $R^{f}$ is one of the most important constraints, i.e., $\rho\left(R^{f}\right)=\rho_{\max }$, by Definition 8 , we have

$$
\rho^{\prime}\left(R^{f}\right)=\rho\left(R^{f}\right) \oslash \rho_{\max }=\rho_{\max } \oslash \rho_{\max }=1 .
$$

Now, in another dynamic situation, where constraints may be added to or removed from a PFCSP, let us examine the proposal that every constraint with the highest priority is forced to be assigned the priority 1 . What if a constraint with a priority higher than that of any existing constraint in the PFCSP is added? What if the constraint with priority 1 is removed from the PFCSP? And even more complicated, what if a constraint with priority 1 is to leave the PFCSP and then after some time return but with a different priority? These might involve recalculating the priorities of all constraints in the PFCSP. However, no such service is provided in the DFP scheme. Thus, users have to reassign priorities to all the constraints again. Clearly, this is inconvenient, especially in the case of a large number of constraints. Instead, our framework overcomes this problem. This is because in our framework there is no maximum value for priorities, and thus we just need to assign a bigger value to the priority of an added constraint if it is considered to be more important than any existing constraint. That is, when we add a new constraint with a priority higher than those of the existing constraints, the new constraint behaves automatically like a hard constraint in the case of complete violation. Similarly, we can understand the situation where some constraints need to be removed (and may come back later).

One example of such a dynamic situation is an automated negotiation system [44] in e-commerce. Here a buyer's requirements on the desired product are expressed as prioritised fuzzy constraints in

\footnotetext{
${ }^{7}$ The DFP scheme uses the scale $[0,1]$ for both priorities and fuzzy constraint membership degrees. This may lead to confusion between the two different concepts. Our framework uses $[0,1]$ only for fuzzy constraint membership, and $[0, \infty)$ for priorities. So, there is no confusion. Notice that in Definition 6 the priority of a constraint takes a value on $[0, \infty)$. Actually, the priority of a constraint could take a value on $[0, a)(a>0)$, e.g., $[0,1)$, since mathematically there is an 1-1 mapping $f:[0, a) \rightarrow[0, \infty)$, i.e.,

$$
f(x)=\frac{1}{a / x-1} .
$$

Unlike the DFP scheme, however, in our framework there is no maximum value for the priority of a constraint, and so the priority of a constraint cannot take a value on $[0, a](a>0)$ since $[0, a]$ implies that the priority of a constraint could take maximum value $a$. This is the reason why we prefer $[0, \infty)$ to $[0,1]$ although there is a $1-1$ mapping from $[0,1)$ to $[0, \infty)$.
} 
Table 5

The country selecting problem based on the DFP scheme

\begin{tabular}{llllll}
\hline & USA & Canada & UK & Australia & Singapore \\
\hline$R_{1}$ & $100 \%$ & $80 \%$ & $90 \%$ & $70 \%$ & $50 \%$ \\
$R_{2}$ & $100 \%$ & $60 \%$ & $80 \%$ & $60 \%$ & $60 \%$ \\
$R_{1} \& R_{2}$ & $100 \%$ & $60 \%$ & $80 \%$ & $60 \%$ & $50 \%$ \\
$R_{3}$ & $40 \%$ & $60 \%$ & $80 \%$ & $60 \%$ & $60 \%$ \\
$R_{1} \& R_{2} \& R_{3}$ & $40 \%$ & $60 \%$ & $80 \%$ & $60 \%$ & $50 \%$ \\
\hline
\end{tabular}

the negotiation agent acting on behalf of the buyer. Firstly, since during the course of a negotiation the agent is autonomous (i.e., the agent gets no instruction from the buyer on running), the buyer must make sure everything is right before running the agent. Thus, it is unavoidable for the buyer to add and remove constraints when testing the agent. Secondly, intuitively a buyer's requirements, i.e., constraints, on the desired product may change over time, and so it is natural that constraints will be added or removed over time. Clearly, it is much more convenient for users if in a scheme of PFCSPs there is a facility which can automatically recalculate the priorities of all constraints in a PFCSP.

Although using the automatic priority recalculation process is more convenient for users, some of them may feel that the process is not transparent to them. However, this may not matter so long as they are satisfied with the final outputs of the system. Of course, we require several more practical applications to check whether our schemes with such a function can produce satisfactory results. Clearly, at the current stage, we cannot answer the question in the paper. As a result, if users care about the transparency more than the convenience, or the convenience is not important (e.g., the size of a PFCSP is small or there are only a few situations where users need to add or remove constraints), they could use their own priority assigning as a kind of priority recalculating process outside the DFP scheme of PFCSPs.

\subsubsection{Non-monotonicity of decision making}

Similar to our system and the FCSP framework, the following example shows that the DFP scheme can also capture the non-monotonicity of decision-making.

Example 19. Suppose the DFP scheme apply to the country choosing problem in Example 14. Clearly, the student can assign the three constraints priorities $0.8,0.6$ and 0.4 , respectively. In the scheme the following formula is used to calculate the local satisfaction degree of a prioritised fuzzy constraint:

$$
\mu_{R^{f}}^{\rho}\left(v_{\operatorname{var}\left(R^{f}\right)}\right)=\max \left\{1-\rho\left(R^{f}\right), \mu_{R^{f}}\left(v_{\operatorname{var}\left(R^{f}\right)}\right)\right\} .
$$

By using the formula we obtain the prioritised satisfaction degrees of these constraints as shown in the second, third and fifth rows of Table 5.

Now we examine the same situation as those of Examples 14 and 15. That is, at the begging the student just considers the first two constraints, and then he further considers the third constraint. From Table 5, we can see clearly that like those of Examples 14 and 15, when he just considers 
the two constraints, the USA is the best choice for him (see the intersection of row 4 and column 2 in Table 5); when he further considers the third constraint, the best choice turns into the UK (see the intersection of row 6 and column 4 in Table 5).

\section{Summary and further work}

The issue of PFCSPs is an important topic in constraint research as we strive to make the model rich enough to model real applications. Dubois et al. introduce the concept of PFCSPs and have made significant contributions to this topic. This paper extends their work in a number of aspects. Firstly, we propose a general axiomatic framework for PFCSPs. Secondly, under our axiomatic framework, we develop a number of methods to construct new schemes for PFCSPs, and use these methods to construct a number of such schemes. Thirdly, by validating our system using examples, we obtain deeper insights into its operation. Fourthly, we compare our priority framework with a weight framework, and reveal that weighted FCSP schemes are the dual of the prioritised FCSP schemes and so can be called posterioritised FCSP schemes. In addition to this, our results show that a PFCSP can be transformed equivalently into an FCSP, and so techniques developed for solving FCSPs can also be adopted for solving PFCSPs.

There are, however, a number of issues that require further investigation:

- Developing alternative and efficient methods, e.g., distributed or agent-based methods, to solve PFCSPs (especially PFCSPs based on general priority T-norms).

- Applying our framework to real applications, e.g., meeting scheduling problems, product selecting problems and negotiation problems in e-commerce.

- Constructing other schemes which obey our axiomatic framework. In particular, with the growing number of schemes the study of their impact on the performance of PFCSP-based systems will become an important issue.

- Discussing further the axioms of global satisfaction degrees. Definition 6 contains five axioms to be met by any global satisfaction degree. As mentioned, the validity of the first axiom can be debated since it may be argued that a fuzzy constraint with the highest priority is not necessarily a hard constraint even when the constraint is completely violated. Giving this, is it possible to define a global satisfaction degree that satisfies only the last four axioms? One such possibility is as follows:

$$
\mu_{R^{f}}^{\rho}\left(v_{\operatorname{var}\left(R^{f}\right)}\right)= \begin{cases}\rho\left(R^{f}\right) \diamond \mu_{R^{f}}\left(v_{\operatorname{var}\left(R^{f}\right)}\right) & \text { if } \exists R_{i}^{f}, R_{j}^{f} \in C^{f}, \rho\left(R_{i}^{f}\right) \neq \rho\left(R_{j}^{f}\right), \\ \mu_{R^{f}}\left(v_{\operatorname{var}\left(R^{f}\right)}\right) & \text { otherwise, }\end{cases}
$$

where $\diamond$ is a priority operator. It would be interesting to study the properties of this definition and look for other possible definitions. In addition, it is also worthwhile checking if there are other axioms that a global satisfaction degree should obey in other contexts. For example, what if in the PFCSP global satisfaction degree formula (45) we replaced the priority T-norm by a general uninorm (e.g., the one given by (54))?

- Applying the idea behind our framework to other topics such as qualitative decision theory, aggregation functions, valued CSPs, semiring-based CSPs, and multi-criteria decision making. (1) Since the DFP scheme $[19,20,23]$ is an application [22,23] of their qualitative decision theory [22-25] 
and this paper extends the DFP scheme, it would be interesting to ascertain whether the idea behind the extension could be reversely applied to extend the qualitative decision theory? (2) Actually, what our axiom system describes is how constraints' priorities and their local satisfaction degree should be aggregated into a global satisfaction degree. Then, what if our axiom system is used as a kind of aggregation operation in other contexts? Since the concept of priority is the dual of weight, it seems that similarly to various kinds of weighted aggregation operation we could define various kinds of prioritised aggregation operation. (3) Qualitative decision theory can be based on fuzzy integrals [24,25,34] and fuzzy integrals can be used as aggregation operators [52]. Then, if our axiom system could be linked to qualitative decision theory and aggregation operators, does it imply that there exists a link between our system and fuzzy integrals? If so, how can the two theories benefit each other? (4) FCSPs are a special case of valued CSPs $[69,16,42,68]$, as well as a special case of semiring-based CSPs [8-10] and prioritised FCSPs are the extension of FCSPs. Then, is it possible to generalise the concepts of prioritised FCSPs to prioritised valued CSPs and semiring-based CSPs? If this is possible, what will happen? (5) In multi-criteria decision making (MCDM), usually one attribute corresponds to a criterion [34,32]; while in CSPs, it is allowed that the combination of multiple attributes corresponds to a constraint. Given this, can we extend MCDM to the situation where it is possible that the combination of multiple attributes corresponds to a criterion? Further, can we introduce the concept of priority into MCDM? Conversely, could some important topics in MCDM, such as the concepts of veto ${ }^{8}$ and favour ${ }^{9}$, be introduced into PFCSPs?

\section{Acknowledgements}

The authors would like to thank the anonymous referees for their comments which significantly improved the quality of the paper.

The work described in this paper was partially supported by a grant from the Research Grants Council of the Hong Kong Special Administrative Region, China (RGC Ref. No. CUHK4304/98E), and the Postdoctoral Fellowship Scheme of the Chinese University of Hong Kong.

\section{References}

[1] J. Aczel, T. Saaty, Procedures for synthesizing ratio judgements, J. Math. Psychol. 27 (1983) 93-102.

[2] R.B. Altman, J.F. Brinkley, Probabilistic constraint satisfaction with structural models: application to organ modeling by radial contours, in: Proc. Ann. Symp. on Computer Applications in Medical Care, 1993, pp. 492-496.

[3] K, Azuma, Weighted sums of certain dependent random variables, Tohoku Math. J. 19 (1967) 357-367.

[4] M. Barbuceanu, W.-K. Lo, A multi-attribute utility theoretic negotiation architecture for electronic commerce, in: Proc. 4th Internat. Conf. on Autonomous Agents, 2000, pp. 239-246.

[5] R.E. Bellman, M. Ciertz, On the analytic formalism of the theory of fuzzy sets, Inform. Sci. 5 (1973) 149-156.

[6] R.E. Bellman, L.A. Zadeh, Decision making in a fuzzy environment, Manage. Sci. P17-B (4) (1970) 141-164.

\footnotetext{
${ }^{8}$ The concept veto means that if the evaluation on a certain criterion is high, it has no effect on the global evaluation, but if it is low, the global evaluation will be low too, whatever the evaluation of the other criteria [52].

${ }^{9}$ The concept favour means that if the evaluation on a certain criterion is low, it has no effect on the global evaluation, but if it is high, the global evaluation will be high too, whatever the evaluation of the other criteria [52].
} 
[7] C. Bessire, Arc-consistency and arc-consistency again, in: Proc. 11th Natl. Conf. on Artificial Intelligence, 1994.

[8] S. Bistarelli, H. Fargier, U. Montanari, F. Rossi, T. Schiex, G. Verfailie, Semiring-based constraint and valued CSPs: frameworks, properties, and comparison, Constraint: Internat. J. 4 (3) (1999) 275-316.

[9] S. Bistarelli, U. Montanari, F. Rossi, Constraint solving over semirings, in: Proc. 14th Internat. Joint Conf. on Artificial Intelligence, 1995, p. 624-630.

[10] S. Bistarelli, U. Montanari, F. Rossi, Semirings-based constraint logic programming, in: Proc. 15th Internat. Joint Conf. on Artificial Intelligence, 1997.

[11] J. Bowen, G. Dozier, Solving randomly generated fuzzy constraint networks using interactive microevolutionary hill-climbing, in: Proc. 1st Internat. Symp. on Soft Computing in Industry, 1996.

[12] N. Bryson, A. Joseph, Generating consensus priority interval vectors for group decision-making in the AHP, J. Multi-Criteria Decision Anal. 9 (2000) 127-137.

[13] C. Carlsson, R. Fullr, Benchmarking in linguistic importance weighted aggregations, Fuzzy Sets and Systems 114 (1) (2000) 35-41.

[14] S. Cost, S. Salzberg, A weighted nearest neighbor algorithm for learning with symbolic features, Machine Learning 10 (1993) 57-78.

[15] R.R. Coifman, R. Fefferman, Weighted norm inequalities for maximal functions and singular integrals, Studia Math. 51 (1974) 241-250.

[16] P. Dago, G. Verfaillie, Nogood recordings for valued constraint satisfaction problems, in: Proc. 8th IEEE Internat. Conf. on Tools with Artificial Intelligence ,1996, pp. 132-139.

[17] D. Dubois, H. Fargier, H. Prade, Flexible constraint satisfaction with application to scheduling problems, Report IRIT/93-30-R, Institute de Recherche en Informatique de Toulouse, 1993.

[18] D. Dubois, H. Fargier, H. Prade, The calculus of fuzzy restrictions as a basis for flexible constraint satisfaction, in: Proc. IEEE Internat. Conf. on Fuzzy Systems, 1993.

[19] D. Dubois, H. Fargier, H. Prade, Propagation and satisfaction of flexible constraints, in: R. Yager, L. Zadeh (ed.), Fuzzy Sets, Neural Networks and Soft Computing, 1994, pp. 166-187.

[20] D. Dubois, H. Fargier, H. Prade, Possibility theory in constraint satisfaction problems: handling priority, preference and uncertainty, Appl. Intelligent 6 (4) (1996) 287-309.

[21] D. Dubois, H. Prade, A class of fuzzy measures based on triangular norms, Internat. J. General Systems 8 (1982) 1.

[22] D. Dubois, H. Prade, Possibility theory as a basis for qualitative decision theory, in: Proc. 14th Internat. Conf. on Artificial Intelligence, 1995, pp. 1924-1930.

[23] D. Dubois, H. Prade, Qualitative possibility theory and its applications to constraint satisfaction and decision under uncertainty, Internat. J. Intelligent Systems 14 (1999) 45-61.

[24] D. Dubois, H. Prade, R. Sabbadin, Decision under qualitative uncertainty with Sugeno integrals: an axiomatic approach, in: Actes du 7eme Congrs Mondial IFSA'97, 1997, pp. 441-446.

[25] D. Dubois, H. Prade, R. Sabbadin, Qualitative decision theory with Sugeno integrals, in: Proc. 14th Conf. on Uncertainty in Artificial Intelligence, 1998, pp. 121-128.

[26] R.O. Duda, P.E. Hart, N.J. Nilsson, R. Reboh, J. Slocum, G. Sutherland, Subjective Bayesian methods for rule-based inference systems, in: AFIPS Conf. Proc., Vol. 45, 1976, pp. 1075-1082.

[27] H. Fargier, J. Lang, Uncertainty in constraint satisfaction problems: a probabilistic approach, in: Proc. European Conf. on Symbolic and Qualitative Approaches to Reasoning and Uncertainty, Lecture Notes in Computer Science, Vol. 747, Springer, Berlin, 1993, pp. 97-104.

[28] J.T. Feddema, C.S.G. Lee, O.R. Mitchell, Weighted selection of image features for resolved rate visual feedback control, IEEE Trans. Robotics Automation 7 (1) (1991) 31-47.

[29] J. Fichtner, On deriving priority vectors from matrices of pairwise comparisons, Socio-Economic Planning Sci. 20 (6) (1986) 399-405.

[30] J. Fodor, Structure of uninorms, Internat. J. Uncertainty, Fuzziness Knowledge-Based Systems 5 (4) (1998) 411427.

[31] M.S. Fox, N. Sedeh, Why is scheduling difficult? A CSP perspective, in: Proc. 9th European Conf. on Artificial Intelligence, 1990, pp. 754-767.

[32] R. Fuller, C. Carlsson, Fuzzy multiple criteria decision making: recent developments, Fuzzy Sets and Systems 78 (1996) 139-153. 
[33] R. Gennari, Soft constraint propagation: generalization and termination conditions, in: Proc. 6th Conf. of Principle and Practice of Constraint Programming, 2000.

[34] M. Grabisch, Fuzzy integral in multicriteria decision making, Fuzzy Sets and Systems 69 (3) (1995) 279-298.

[35] R.H. Guttman, P. Maes, Agent-mediated integrative negotiation for retail electronic commerce, in: P. Noriega, C. Sierra (Eds.), Agent Mediated Electronic Commerce, Lecture Notes in Artificial Intelligence, Vol. 1571, Springer, Berlin, 1998, pp. 70-90.

[36] T.H. Hong, A. Rosenfeld, Compact region extraction using weighted pixel linking in a pyramid, IEEE Trans. on Pattern Anal. Machine Intelligence 6 (2) (1984) 222-229.

[37] R.W.L. Kam, J.H.-M. Lee, Fuzzifying the constraint hierarchies framework, in: Proc. 4th Internat. Conf. on Principles and Practices of Constraint Programming, Lecture Notes in Artificial Intelligence, Vol. 1520, Springer, Berlin, 1998, pp. 280-294.

[38] E.P. Klement, R. Mesiar, E. Pap, On the relationship of associative compensatory operators to triangular norms and conorms, Internat. J. Uncertainty, Fuzziness Knowledge-Based Systems 4 (2) (1996) 129-144.

[39] R. Kowalczyk, On negotiation as a distributed fuzzy constraint satisfaction problem, in: Proc. 3rd Internat. Symp. on Soft Computing for Industry of the World Automation Congress, 2000, pp. 631-637.

[40] R. Kowalczyk, V. Bui, On fuzzy e-negotiation agents: autonomous negotiation with incomplete and imprecise information, in: Proc. DEXA e-Negation Workshop, 2000.

[41] R. Kowalczyk, V. Bui, On constraint-based reasoning in e-negotiation agents, in: F. Dignum, U. Cortés (Eds.), Agent Mediated E-commerce III, Lecture Notes in Artificial Intelligence, Vol. 2003, Springer, Berlin, 2000, pp. 31-46.

[42] M. Lemaitre, G. Verfaillie, An incomplete method for solving distributed valued constraint satisfaction problems, in: Proc. AAAI97 Workshop on "Constraints and Agents" Providence, RI, USA, 1997.

[43] Y.-M. Li, Z.-K. Shi, Remarks on uninorm aggregation operators, Fuzzy Sets and Systems 114 (2000) 377-380.

[44] X. Luo, N.R. Jennings, N.R. Shadbolt, H.-F. Leung, J.H.-H. Lee, A fuzzy constraint based model for bilateral, multi-issue negotiations in semi-competitive environments, Artificial Intelligence, submitted.

[45] X. Luo, H.-F. Leung, J.H.-M. Lee, Theory and properties of a selfish protocol for multi-agent meeting scheduling using fuzzy constraints, in: Proc. 14th European Conf. on Artificial Intelligence, 2000, pp. 373-377.

[46] X. Luo, C. Zhang, An axiom foundation for uncertain reasonings in rule-based expert systems: NT-algebra, Knowledge Inform. Systems: Internat. J. 1 (4) (1999) 415-433.

[47] X. Luo, C. Zhang, Isomorphic transformations of uncertainties for incorporating EMYCIN-style and PROSPECTOR-style systems into a distributed expert system, J. Comput. Sci. Technol. 14 (4) (1999) 368-392.

[48] X. Luo, C. Zhang, J. Cai, The weighting issue in fuzzy logic, Informat.: Internat. J. Comput. Inform. 21 (1997) $255-262$.

[49] X. Luo, C. Zhang, H.-F. Leung, A class of isomorphic transformations for integrating EMYCIN-style and PROSPECTOR-style systems into a rule-based multi-agent system, in: H. Nakashima, C. Zhang (Ed.), Approaches to Intelligent Agents, Lecture Notes in Artificial Intelligence, Vol. 1733, Springer, Berlin, 1999, pp. 211-225.

[50] X. Luo, C. Zhang, H.-F. Leung, Information sharing between heterogeneous uncertain reasoning models in a multi-agent environment: a case study, Internat. J. Approximate Reasoning 27 (1) (2001) 27-59.

[51] A.K. Mackworth, Consistency in networks of relations, Artificial Intelligence 8 (1) (1977) 99-118.

[52] J.-L. Marichal, On Sugeno integral as an aggregation function, Fuzzy Sets and Systems 114 (3) (2000) 347-365.

[53] J. McCarthy, Circumscriptions: a form of non-monotonic reasoning, Artificial Intelligence 13 (1980) 27-39.

[54] P. Meseguer, J. Larrosa, Solving fuzzy constraint satisfaction problems, in: Proc. 6th IEEE Internat. Conf. on Fuzzy Systems, Vol. 3, 1997, pp. 1233-1238.

[55] R. Mohr, T. Henderson, Arc and path consistency revisited, Artificial Intelligence 28 (1986) 225-233.

[56] E. Motta, Reusable Components for Knowledge Modelling: Case Studies in Parametric Design Problem Solving, IOS Press, Berlin, 1999.

[57] C.V. Negoita, Management applications of system theory, Editura Tehnica, Bucharest and Birkhäuser Verlag, Basel, 1979.

[58] J. Peasall (Ed.), The new Oxford dictionary of English, Oxford University Press, Oxford, 2001.

[59] A.D. Pierro, H. Wiklicky, On probabilistic CCP, in: Proc. APPPIA-GULP-PRODE'97 Joint Conf. on Declarative Programming, June 1997.

[60] A.D. Pierro, H. Wiklicky, An operational semantics for probabilistic concurrent constraint programming, in: Proc. Internat. Conf. on Computer Language, 1998, pp. 174-183. 
[61] S. Raughunathan, A planning aid: an intelligent modelling system for planning problems based on constraint satisfaction, IEEE Trans. on Knowledge Data Eng. 4 (4) (1992) 317-335.

[62] R. Reiter, A logic for default reasoning, Artificial Intelligence 13 (1-2) (1980) 81-132.

[63] A. Rosenfeld, R. Hummel, S. Zucker, Scene labelling by relaxation operations, IEEE Trans. on Systems, Man Cybernet. 6 (6) (1976) 420-433.

[64] Z. Ruttkay, Fuzzy constraint satisfaction, in: Proc. IEEE Internat. Conf. on Fuzzy Systems, 1994.

[65] T. Saaty, The Analytic Hierarchy Process, McGraw-Hill, New York, 1980.

[66] R. Sabbadin, D. Dubois, H. Prade, A fuzzy constraint satisfaction problem in the wine industry, J. Intelligent Fuzzy Systems 6 (1998) 361-374.

[67] T.W. Sandholm, Distributed rational decision making, in: G. Weiss (Ed.), Multiagent Systems: A Modern Approach to Distributed Artificial Intelligence, The MIT Press, Cambridge, MA, 1999, pp. 201-258.

[68] T. Schiex, Valued constraint networks. in: Proc. 6th Conf. on Principles and Practice of Constraint Programming, 2000.

[69] T. Schiex, H. Fargier, G. Verfaillie, Valued constraint satisfaction problems: hard and easy problems, in: Proc. Internat. Joint Conf. on Artificial Intelligence, 1995, pp. 631-637.

[70] G. Schreiber, H. Akkermans, A. Anjewierden, R.d. Hoog, N. Shadbolt, W.V.d. Velde, B. Wielinga, Knowledge Engineering and Management: The CommonKADS Methodology, The MIT Press, Cambridge, MA, 1999, pp. 167186 (Chapter 5).

[71] B. Schweizer, A. Sklar, Associative functions and abstract semigroups, Publ. Math. Debrecen 10 (1963) 69-81.

[72] N.M. Shazeer, M.L. Littman, G.A. Keim, Solving crossword puzzles as probabilistic constraint satisfaction, in: Proc. 16th Natl. Conf. on Artificial Intelligence, 1999, pp. 156-162.

[73] R. Sun, B. Chu, R. Wilhelm, J. Yao, A CSP-based model for integrated supply chain, in: Proc. AAAI-99 Workshop on AI for Electronic Commerce, 1999.

[74] E.P.K. Tsang, Foundations of Constraint Satisfaction, Academic Press, New York, 1993.

[75] T. Tsuruta, T. Shintani, Scheduling meeting using distributed valued constraint satisfaction algorithm, in: Proc. 14th European Conf. on Artificial Intelligence, 2000, pp. 383-387.

[76] J.H.Y. Wong, H.-F. Leung, Solving fuzzy constraint satisfaction problems with fuzzy GENET, in: Proc. 10th Internat. Conf. on Tools with Artificial Intelligence, 1998, pp. 184-191.

[77] J.H.Y. Wong, H.-F. Leung, Extending GENET to solve fuzzy constraint satisfaction problems. Proc. AAAI-98, 1998, pp. 380-385.

[78] R.R. Yager, Misrepresentations and challenges: a response to Elkan, IEEE Expert, August 1994, pp. 41-42.

[79] R.R. Yager, A. Rybalov, Uninorm aggregation operators, Fuzzy Sets and Systems 80 (1996) 111-120.

[80] R.R. Yager, A. Rybalov, Uni-norms: a unification of T-norm and T-conorms, in: Proc. Nafips Conf., 1996, pp. 50-54.

[81] L.A. Zadeh, Fuzzy sets, Inform. Control 8 (1965) 338-353.

[82] C. Zhang, X. Luo, Isomorphic transformation of uncertainties of propositions among the EMYCIN and PROSPECTOR uncertain models, in: Proc. 2nd Internat. Conf. on Multi-Agent Systems, 1996, p. 465.

[83] H.-J. Zimmermann, Fuzzy programming and linear programming with several objective function, Fuzzy Sets and Systems 1 (1978) 45-55.

[84] H.-J. Zimmermann, Fuzzy Set Theory and Its Applications, 3rd ed., Kluwer Academic Publishers, Dordrecht, 1996. 